\title{
Rates of Convex Approximation in Non-Hilbert Spaces
}

\author{
Michael J. Donahue \\ Leonid Gurvits \\ Christian Darken \\ Eduardo Sontag
}

August 23, 1994

\begin{abstract}
This paper deals with sparse approximations by means of convex combinations of elements from a predetermined "basis" subset $S$ of a function space. Specifically, the focus is on the rate at which the lowest achievable error can be reduced as larger subsets of $S$ are allowed when constructing an approximant. The new results extend those given for Hilbert spaces by Jones and Barron, including in particular a computationally attractive incremental approximation scheme. Bounds are derived for broad classes of Banach spaces; in particular, for $L_{p}$ spaces with $1<p<\infty$, the $O\left(n^{-1 / 2}\right)$ bounds of Barron and Jones are recovered when $p=2$.

One motivation for the questions studied here arises from the area of "artificial neural networks," where the problem can be stated in terms of the growth in the number of "neurons" (the elements of $S$ ) needed in order to achieve a desired error rate. The focus on non-Hilbert spaces is due to the desire to understand approximation in the more "robust" (resistant to exemplar noise) $L_{p}, 1 \leq p<2$ norms.

The techniques used borrow from results regarding moduli of smoothness in functional analysis as well as from the theory of stochastic processes on function spaces.
\end{abstract}

\section{Introduction}

The subject of this paper concerns the problem of approximating elements of a Banach space $X$-typically presented as a space of functions-by means of finite linear combinations of elements from a predetermined subset $S$ of $X$. In contrast to classical linear approximation techniques, where optimal approximation is desired and no penalty is imposed on the number of elements used, we are interested here in sparse approximants, that is to say, combinations that employ few elements. In particular, we are interested in understanding the rate at which the achievable error can be reduced as one increases the number allowed. Such 
questions are of obvious interest in areas such as signal representation, numerical analysis, and neural networks (see below).

Rather than arbitrary linear combinations $\sum_{i} a_{i} g_{i}$, with $a_{i}$ 's real and $g_{i}$ 's in $S$, it turns out to be easier to understand approximations in terms of combinations that are subject to a prescribed upper bound on the total coefficient sum $\sum_{i}\left|a_{i}\right|$. After normalizing $S$ and replacing it by $S \cup-S$, one is led to studying approximations in terms of convex combinations. This is the focus of the current work.

To explain the known results and our new contributions, we first introduce some notation.

\subsection{Optimal Approximants}

Let $X$ be a Banach space, with norm $\|\cdot\|$. Take any subset $S \subseteq X$. For each positive integer $n$, we let $\operatorname{lin}_{n} S$ consist of all sums $\sum_{i=1}^{n} a_{i} g_{i}$, with $g_{1}, \ldots, g_{n}$ in $S$ and with arbitrary real numbers $a_{1}, \ldots, a_{n}$, while we let $\operatorname{co}_{n} S$ be the set of such sums with the constraint that all $a_{i} \in[0,1]$ and $\sum_{i} a_{i}=1$. The distances from an element $f \in X$ to these spaces are denoted respectively by

$$
\left\|\operatorname{lin}_{n} S-f\right\|:=\inf \left\{\|h-f\|, h \in \operatorname{lin}_{n} S\right\}
$$

and

$$
\left\|\mathrm{co}_{n} S-f\right\|:=\inf \left\{\|h-f\|, h \in \mathrm{co}_{n} S\right\} .
$$

Of course, always $\left\|\operatorname{lin}_{n} S-f\right\| \leq\left\|\operatorname{co}_{n} S-f\right\|$. For each subset $S \subseteq X$, $\operatorname{lin} S=$ $\cup_{n} \operatorname{lin}_{n} S$ and $\operatorname{co} S=\cup_{n} \operatorname{co}_{n} S$ denote respectively the linear span and the convex hull of $S$. We use bars to denote closure in $X$; thus, for instance, $\overline{\cos }$ is the closed convex hull of $S$. Note that saying that $f \in \overline{\operatorname{lin} S}$ or $f \in \overline{\operatorname{coS}}$ is the same as saying that $\lim _{n \rightarrow+\infty}\left\|\operatorname{lin}_{n} S-f\right\|=0$ and $\lim _{n \rightarrow+\infty}\left\|\operatorname{co}_{n} S-f\right\|=0$ respectively; in this case, we say for short that $f$ is (linearly or convexly) approximable by $S$. These distances as a function of $n$ represent the convergence rates of the best approximants to the target function $f$. The study of such rates is standard in approximation theory (e.g., Powell [23]), but the questions addressed here are not among those classically considered.

Let $\phi$ be a positive function on the integers. We say that the space $X$ admits a (convex) approximation rate $\phi(n)$ if for each bounded subset $S$ of $X$ and each $f \in \overline{\operatorname{coS} S},\left\|\mathrm{co}_{n} S-f\right\|=O(\phi(n))$. (The constant in this estimate is allowed to, and in general will, depend on $S$, typically through an upper bound on the norm of elements of $S$.) One could of course also define the analogous linear approximation rates; we do not do so because at this time we have no nontrivial results to report in that regard. (The implications of the restriction to convex approximates is examined in Appendix A.)

Jones [15] and Barron [2] showed that every Hilbert space admits an approximation rate $\phi(n)=1 / \sqrt{n}$. One of our objectives is the study of such rates for non-Hilbert spaces. To date the larger issue of convergence rates in more 
general Banach spaces and in the important subclass of $L_{p}, p \neq 2$, spaces has not been addressed. Barron [3] showed that the same rate is obtained in the uniform norm, but only for approximation with respect to a particular class of sets $S$.)

\subsection{Incremental Approximants}

Jones [15] considered the procedure of constructing approximants to $f$ incrementally, by forming a convex combination of the last approximant with a single new element of $S$; in this case, the convergence rate in $L_{2}$ is interestingly again $O(1 / \sqrt{n})$. Incremental approximants are especially attractive from a computational point of view. In the neural network context, they correspond to adding one "neuron" at a time to decrease the residual error. We next define these concepts precisely.

Again let $X$ be a Banach space with norm $\|\cdot\|$. Let $S \subseteq X$. An incremental sequence (for approximation in $\operatorname{co} S$ ) is any sequence $f_{1}, f_{2}, \ldots$ of elements of $X$ so that $f_{1} \in S$ and for each $n \geq 1$ there is some $g_{n} \in S$ so that $f_{n+1} \in$ $\operatorname{co}\left(\left\{f_{n}, g_{n}\right\}\right)$.

We say that an incremental sequence $f_{1}, f_{2}, \ldots$ is greedy (with respect to $f \in \overline{\cos }$ ) if

$$
\left\|f_{n+1}-f\right\|=\inf \left\{\|h-f\| \mid h \in \operatorname{co}\left(\left\{f_{n}, g\right\}\right), g \in S\right\}, n=1,2, \ldots
$$

The set $S$ is generally not compact, so we cannot expect the infimum to be attained. Given a positive sequence $\epsilon=\left(\epsilon_{1}, \epsilon_{2}, \ldots\right)$ of allowed "slack" terms, we say that an incremental sequence $f_{1}, f_{2}, \ldots$ is $\varepsilon$-greedy (with respect to $f$ ) if

$$
\left\|f_{n+1}-f\right\|<\inf \left\{\|h-f\| \mid h \in \operatorname{co}\left(\left\{f_{n}, g\right\}\right), g \in S\right\}+\varepsilon_{n}, \quad n=1,2, \ldots .
$$

Let $\phi$ be a positive function on the integers. We say that $S$ has an incremental (convex) scheme with rate $\phi(n)$ if there is an incremental schedule $\varepsilon$ such that, for each $f$ in $\overline{\operatorname{coS} S}$ and each $\varepsilon$-greedy incremental sequence $f_{1}, f_{2}, \ldots$, it holds that

$$
\left\|f_{n}-f\right\|=O(\phi(n))
$$

as $n \rightarrow+\infty$. Finally, we say that the space $X$ admits incremental (convex) schemes with rate $\phi(n)$ if every bounded subset $S$ of $X$ has an incremental scheme with rate $\phi(n)$.

The intuitive idea behind this definition is that at each stage we attempt to obtain the best approximant in the restricted subclass consisting of convex combinations $\left(1-\lambda_{n}\right) f_{n}+\lambda_{n} g$, with $\lambda_{n}$ in $[0,1], g$ in $S$, and $f_{n}$ being the previous approximant. It is also possible to select the sequence $\lambda_{1}, \lambda_{2}, \ldots$ beforehand. We say that an incremental sequence $f_{1}, f_{2}, \ldots$ is $\epsilon$-greedy (with respect to $f$ ) with convexity schedule $\lambda_{1}, \lambda_{2}, \ldots$ if

$$
\left\|f_{n+1}-f\right\|<\inf \left\{\left\|\left(\left(1-\lambda_{n}\right) f_{n}+\lambda_{n} g\right)-f\right\| \mid g \in S\right\}+\varepsilon_{n}, \quad n=1,2, \ldots
$$


Table 1: The order of the worst-case rate of approximation in $L_{p}$. "No" means that the approximants do not converge in the worst case.

\begin{tabular}{|c|c|c|c|c|}
\hline $\mathrm{p}$ & 1 & $(1,2)$ & {$[2, \infty)$} & $\infty$ \\
\hline optimal & 1 & $n^{1 / p-1}$ & $n^{-1 / 2}$ & 1 \\
incremental & No & $n^{1 / p-1}$ & $n^{-1 / 2}$ & No \\
\hline
\end{tabular}

One could also define the analogous linear incremental schemes, for which one does not require $\lambda_{n} \in[0,1]$, but, as before, we only report results for the convex case.

Informally, from now on we refer to the rates for convex approximation as "optimal rates" and use the terminology "incremental rates" for the best possible rates for incremental schemes. For any incremental sequence, $f_{n} \in$ $\mathrm{co}_{n}(S)$, so clearly optimal rates are always majorized by the corresponding incremental rates.

The main objective of this paper ${ }^{1}$ is to analyze both optimal and incremental rates in broad classes of Banach spaces, specifically including $L_{p}, 1 \leq p \leq \infty$. A summary of our rate bounds for the special case of the spaces $L_{p}$ is given as Table 1. In general, we find that the worst-case rate of approximation in the "robust" $L_{p}, 1 \leq p<2$, norms is worse than that in $L_{2}$, unless some additional conditions are imposed on the set $S$.

\subsection{Neural Nets}

The problem is of general interest, but we were originally motivated by applications to "artificial neural networks." In that context the set $S$ is typically of the form

$$
S=\left\{g: \mathbb{R}^{d} \rightarrow \mathbb{R} \mid \exists a \in \mathbb{R}^{d}, b \in \mathbb{R}, \text { s.t. } g(x)= \pm \sigma(a \cdot x+b)\right\},
$$

where $\sigma: \mathbb{R} \rightarrow \mathbb{R}$ is a fixed function, called the activation or response function. Typically, $\sigma$ is a smooth "sigmoidal" function such as the logistic function $\left(1+e^{-x}\right)^{-1}$, but it can be discontinuous, such as the Heaviside function (the characteristic function of $[0, \infty)$ ). The elements of $\operatorname{lin}_{n} S$ are called single hidden layer neural networks (with activation $\sigma$ and a linear output layer) with $n$ hidden units. For neural networks, then, the question that we investigate translates into the study of how the approximation error scales with the number of hidden units in the network.

Neural net approximation is a technique widely used in empirical studies. Mathematically, this is justified by the fact that, for each compact subset $M$ of

\footnotetext{
${ }^{1}$ A preliminary version of some of the results presented in this paper appeared as (Darken, Donahue, Gurvits and Sontag [6]).
} 
$\mathbb{R}^{d}$, restricting elements of $S$ to $M$, one has that $\overline{\operatorname{lin} S}=C^{0}(M)$, that is, $\operatorname{lin} S$ is dense in the set of continuous functions under uniform convergence (and hence also in most other function spaces). This density result holds under extremely weak conditions on $\sigma$; being locally Riemann integrable and non-polynomial is enough. See for instance (Leshno et al., [19]).

Spaces $L_{p}$ with $p$ equal to or slightly greater than one are particularly important because of their usefulness for robust estimation (e.g., Rey [24]). In the particular context of regression with neural networks, Hanson [13] presents experimental results showing the superiority of $L_{p}(p \ll 2)$ to $L_{2}$.

\subsection{Connections to Learning Theory}

Of course, neural networks are closely associated with learning theory. Let us imagine that we are attempting to learn a target function that lies in the convex closure of a predetermined set of basis functions $S$. Our learned estimate of the target function will be represented as a convex combination of a subset of $S$. For each $n$ in an increasing sequence of values of $n$, we optimize our choice of basis functions and their convex weighting over a sufficiently large data set (the size of which may depend on $n$ ). Let us assume that the problem is "learnable", e.g., that over the class of probability measures of interest on the domain of the functions in $S$, the difference between one's estimates of the error based on examples must converge to the true error uniformly over all possible approximants. Then the generalization error (expected loss over the true exemplar distribution) must go to zero at least as fast as the order of the upper bounds in this work. Thus our bounds represent a guarantee of how fast generalization error will decrease in the limiting case when exemplars are so cheap that we do not care how many we use during training.

Moreover, since for error functions that are $L_{p}$ norms our bounds are tight, we can say something even stronger in this case. For $L_{p}$, there exists a set of basis functions and a function in their convex hull such that no matter how many examples are used in training, the error can decrease no faster than the bounds we have provided. Thus, our results exhibit a worst-case speed limitation for learning.

\subsection{Contents of the Paper}

It is a triviality that optimal approximants to approximable functions always converge. However, the rates of convergence depend critically upon the structure of the space. In some spaces, like $L_{1}$, there exist target functions for which the rate can be made arbitrarily slow (Sect. 2.1). In Banach spaces of (Rademacher) type $t$ with $t>1$, however, a rate bound of $O\left(n^{-(t-1)}\right)$ is obtained (Sect. 2.2). For $L_{p}$ spaces these results specialize to those of Table 1. Particular examples of $L_{p}$ spaces are given to show that the orders given in our bounds cannot in general be sharpened (Sect. 2.3). 
Section 3 studies incremental approximation. A particularly interesting aspect of these results is that the new element of $S$ added to the incremental approximant is not required to be the best possible choice. Instead, the new element can meet a less stringent test (Theorem 3.5). Also, the convex combination of the elements included in the approximant is not optimized. Instead a simple average is used. (This is an example of a fixed convexity schedule, as defined in Sect. 1.2.) Thus, our incremental approximants are the simplest yet studied, simpler even than those of Jones [15]. Nonetheless, the same worst-case order is obtained for these approximants on $L_{p}, 1<p<\infty$, as for the optimal approximant. In more general spaces, the incremental approximants may not even converge (Sect. 3.1). However, if the space has a modulus of smoothness of power type greater than one, or is of Rademacher type $t$, then rate bounds can be given (Sects. 3.2 and 3.3).

Both optimal and incremental convergence rates may be improved if $S$ has special structure (Sect. 4). In particular, we provide some analysis of the situation where $S$ is a finite-VC dimension set of indicator functions and the sup norm is to be used (Sect. 4.2), which is a common setting for neural network approximation.

\section{Optimal Approximants}

In this section we study rates of convergence for optimal convex approximates. To illustrate the fact that the issue is nontrivial, we begin by identifying a class of spaces for which the best possible rate $\phi(n)$ is constant, that is to say, no nontrivial rate is possible (Theorem 2.3). This class includes infinite dimensional $L_{1}$ and $L_{\infty}($ or $C(X))$ spaces.

In Theorem 2.8 we study general bounds valid for spaces of (Rademacher) type $t$. It is well-known that $L_{p}$ spaces with $1 \leq p<\infty$ are of type $\min \{p, 2\}$ (Ledoux and Talagrand [18]); on this basis an explicit specialization to $L_{p}$ is given in (10).

We then close this section with explicit examples showing that the obtained bounds are tight.

\subsection{Examples Of Spaces Where No Rate Bound Is Possi- ble}

In some spaces, the worst-case rate of convergence of optimal approximants can be shown to be arbitrarily slow.

Lemma 2.1 Let $\left(a_{n}\right)$ be a positive, convex $\left(a_{n}+a_{n+2} \geq 2 a_{n+1}\right)$ sequence converging to 0 . Define $a_{0}=2 a_{1}$ and $b_{n}=a_{n-1}-a_{n}$. Let $S=\left\{a_{0} e_{k}\right\}$, where $\left\{e_{k}\right\}$ is the canonical basis in $l_{1}$, and consider $f=\left(b_{n}\right)$ as an element of $l_{1}$. Then $f \in \overline{\operatorname{coS}}$ and

$$
\left\|\operatorname{lin}_{N} S-f\right\|=a_{N} \quad \text { for all } N
$$


Proof: Note that $\sum_{n=1}^{\infty} b_{n} / a_{0}=1$, so clearly $f \in \overline{\operatorname{coS} S}$. By convexity $\left(b_{n}\right)$ is a non-increasing sequence, so

$$
\left\|\operatorname{lin}_{N} S-f\right\|=\sum_{i=N+1}^{\infty} b_{i}=\sum_{i=N+1}^{\infty} a_{i-1}-a_{i}=a_{N} .
$$

Consider next the space $l_{\infty}$. Let $\epsilon_{k}$ be an enumeration of all $\{-1,0,1\}$-valued sequences that are eventually constant, i.e., $\epsilon_{k}(n) \in\{-1,0,1\}$ for all $n \in \mathbb{N}$, and for each $k$ there exists an $N$ such that $\epsilon_{k}(n)=\epsilon_{k}(N)$ for all $n>N$. For each $n$, let $g_{n} \in l_{\infty}$ be the sequence $g_{n}(k)=\epsilon_{k}(n)$, and define the map $T: l_{1} \rightarrow l_{\infty}$ by $T\left(e_{n}\right)=g_{n}$. The reader may check that $T$ is an isometric embedding. Therefore $T$ carries the example of Lemma 2.1 into $l_{\infty}$.

What happens in $c_{0}$, the space of all sequences converging to 0 ? We will now construct a projection from $T\left(l_{1}\right)$ into $c_{0}$ that will retain the desired convergence rate. We will need, however, the extra restriction that the sequence $\left(a_{n}\right)$ be strictly convex, i.e., that $a_{n}+a_{n+2}>2 a_{n+1}$.

Let $b_{n}=a_{n-1}-a_{n}$ as before, and define the auxiliary sequences

$$
\begin{aligned}
& \underline{c}_{N}=\min \left\{n \in \mathbb{N} \mid b_{n}<a_{N}\right\} \\
& \bar{c}_{N}=\min \left\{n \in \mathbb{N} \mid n>N+1 \text { and } a_{n}<b_{N}-b_{N+1}\right\} .
\end{aligned}
$$

The sequence $\underline{c}$ is well defined because $a_{N}>0$ for all $N$ and $b_{n} \downarrow 0$. Similarly, $\bar{c}$ is well defined since $a_{n} \downarrow 0$ and by strict convexity $b_{N}-b_{N+1}>0$. Note that $\underline{c}_{N} \leq N+1<N+2 \leq \bar{c}_{N}$. Moreover, $\underline{c}_{N}$ (and hence $\bar{c}_{N}$ ) goes to infinity with $N$ since $b_{n}>0$ for each $n$ while $a_{N} \downarrow 0$.

Next define for each $N \in \mathbb{N}$,

$$
A_{N}=\left\{k \in \mathbb{N} \mid \epsilon_{k}(n)=0 \text { for } n<\underline{c}_{N} \text { and } \epsilon_{k}(n)=\epsilon_{k}\left(\bar{c}_{N}+1\right) \text { for } n>\bar{c}_{N}\right\},
$$

and define for convenience the single element set $A_{0}=\left\{k \mid \epsilon_{k}=\delta_{k}\right\}$, where $\delta_{k}(n)$ is the sequence that is 1 for $n=k$ and 0 otherwise. Then let $A=\cup_{N} A_{N}$.

Let $P$ be the projection that sends an element $h$ of $l_{\infty}$ to the sequence $P(h)(k)=h(k)$ if $k \in A$ and $P(h)(k)=0$ otherwise. Notice that if $\epsilon_{k}(n) \neq 0$, then $k \notin A_{N}$ for all $N$ such that $n<\underline{c}_{N}$. Since $\underline{c}_{N} \rightarrow \infty$, if follows that there exists for each $n$ only finitely many $k$ 's in $A$ such that $\epsilon_{k}(n) \neq 0$. (Each $A_{N}$ is a finite set.) Therefore $P\left(g_{n}\right)=P \circ T\left(e_{n}\right) \in c_{0}$ for each $n$, i.e., $P \circ T: l_{1} \rightarrow c_{0}$.

It remains to show that

$$
\left\|P \circ T(f)-\operatorname{lin}_{N} P \circ T(S)\right\|=a_{N}
$$

Let us introduce the notation $\tilde{h}$ for $P \circ T(h), h \in l_{1}$, and similarly $\tilde{S}$ for $P \circ T(S)$. It is clear that $\left\|\tilde{f}-\operatorname{lin}_{N} \tilde{S}\right\| \leq a_{N}$, since $T$ is an isometry and $\|P\|=1$. To examine the bound from below, let $\tilde{f}_{N}=\sum_{n=1}^{N} d_{n} \tilde{e}_{m_{n}}$ be an arbitrary element 
of $\operatorname{lin}_{N} \tilde{S}$, where $\left\{m_{1}, m_{2}, \ldots, m_{N}\right\}$ is a sampling of $\mathbb{N}$ of size $N$. We aim to produce a $k_{0} \in A$ such that $\tilde{f}_{N}\left(k_{0}\right)=0$ and $\tilde{f}\left(k_{0}\right) \geq a_{N}$, since then

$$
|| \tilde{f}-\tilde{f}_{N}||=\sup _{k \in A}\left|\tilde{f}(k)-\tilde{f}_{N}(k)\right| \geq\left|\tilde{f}\left(k_{0}\right)-\tilde{f}_{N}\left(k_{0}\right)\right| \geq a_{N} .
$$

Let $n_{0}=\min \left(\mathbb{N} \backslash\left\{m_{1}, m_{2}, \ldots, m_{N}\right\}\right)$. If $n_{0}<\underline{c}_{N}$, select $k_{0}$ such that $\epsilon_{k_{0}}=\delta_{n_{0}}$. If $n_{0}=N+1$ (which is the largest possible value for $n_{0}$ ), select $k_{0}$ such that $\epsilon_{k_{0}}(n)=0$ for $n \leq N$ and $=1$ otherwise. It follows from $\underline{c}_{N} \leq N+1$ that $k_{0} \in A$ in either event, and clearly $\tilde{f}_{N}\left(k_{0}\right)=0$. Moreover, in the first case $\tilde{f}\left(k_{0}\right)=b_{n_{0}} \geq a_{N}$ by the definition of $\underline{c}_{N}$, while in the second case, $\tilde{f}\left(k_{0}\right)=$ $\sum_{n=N+1}^{\infty} b_{n}=a_{N}$.

Lastly, consider the case $\underline{c}_{N} \leq n_{0} \leq N$. Select $k_{0}$ so that $\epsilon_{k_{0}}(n)=0$ if $n \in\left\{m_{1}, m_{2}, \ldots, m_{N}\right\}$ or if $n>\bar{c}_{N}$, and $\epsilon_{k_{0}}(n)=1$ otherwise. This sequence is guaranteed to be in $A_{N}$, and $\tilde{f}_{N}\left(k_{0}\right)=0$. Moreover,

$$
\tilde{f}\left(k_{0}\right)=\sum_{n=\underline{c}_{N}}^{\bar{c}_{N}} b_{n} \epsilon_{k_{0}}(n) \geq b_{N}+\sum_{n=N+2}^{\bar{c}_{N}} b_{n} .
$$

The inequality holds because $\epsilon_{k_{0}}(n)=1$ for at least one $n \leq N$, and $\left(b_{n}\right)$ is a decreasing sequence. It then follows from the definition of $\bar{c}_{N}$ that

$$
b_{N}+\sum_{n=N+2}^{\bar{c}_{N}} b_{n}=b_{N}+a_{N+1}-a_{\bar{c}_{N}}>a_{N},
$$

so $\left\|\tilde{f}-\tilde{f}_{N}\right\| \geq a_{N}$, completing the proof.

Lemma 2.2 Let $\left(a_{n}\right)$ be a positive, strictly convex $\left(a_{n}+a_{n+2}>2 a_{n+1}\right)$ sequence converging to 0 . Then there exists a bounded set $S \subset c_{0}$ (with $\|g\| \leq 2 a_{1}$ for all $g \in S$ ) and $f \in \overline{\operatorname{coS}}$ such that

$$
\left\|f-\operatorname{lin}_{N} S\right\|=a_{N} \quad \text { for all } N \in \mathbb{N} .
$$

An alternate method of proof is to replace the projection $P$ in the discussion above with a map $T^{\prime}: l_{\infty} \rightarrow c_{0}$ defined by $T^{\prime}(h)(k)=\delta_{k} h(k)$, where $\delta_{k} \downarrow 0$ is carefully chosen (as a function of $\left(a_{n}\right)$ ) to preserve the inequality $\| T^{\prime} \circ T(f)-$ $\operatorname{lin}_{N} T^{\prime} \circ T(S) \| \geq a_{N}$. The details are left to the reader.

In either method, the constructed base set $S \subset c_{0}$ depends on the rate sequence $\left(a_{n}\right)$. It is interesting to compare this with the situation in $l_{1}$ and $l_{\infty}$, where the set $S$ is universal, i.e., independent of $\left(a_{n}\right)$. (Though the limit function $f \in \overline{\operatorname{coS}}$ does vary with $\left(a_{n}\right)$.)

The preceding discussion showing the absence of a rate bound in $l_{\infty}$ relied upon an isometric embedding of $l_{1}$ into $l_{\infty}$. This argument can of course be extended to other spaces, and in fact it suffices to have an isomorphic embedding, i.e., a bounded linear map with bounded inverse. 
Theorem 2.3 Let $X$ be a Banach space with a subspace isomorphic to either $l_{1}$ or $c_{0}$. Then for any positive sequence $\left(a_{n}\right)$ converging to 0 , it is possible to construct a bounded set $S$ and $f \in \overline{\operatorname{coS}}$ such that

$$
\left\|\operatorname{co}_{N} S-f\right\| \geq\left\|\operatorname{lin}_{N} S-f\right\| \geq a_{N} .
$$

Proof: If $\left(a_{n}\right)$ is not convex, then replace it with a convex sequence $\left(\bar{a}_{n}\right)$ such that $\bar{a}_{n} \geq a_{n}$ for all $n$. This is a well-known construction. See, for example, (Stromberg [28], p. 515). In the $c_{0}$ case one may also substitute $\left(\bar{a}_{n}+1 / n\right)$ for $\left(\bar{a}_{n}\right)$, if necessary, to make the sequence strictly convex.

The first inequality follows immediately from the definitions of $\operatorname{co}_{N}$ and $\operatorname{lin}_{N}$, so it suffices to show $\left\|\operatorname{lin}_{N} S-f\right\| \geq a_{N}$. To do this, construct $S$ and $f$ in $l_{1}$ or $c_{0}$ via Lemma 2.1 or 2.2 , replacing $\left(a_{n}\right)$ with $\left(\left\|T^{-1}\right\| a_{n}\right)$, where $T$ is the postulated isomorphism. Then use $T$ to transfer the example back into $X$.

Corollary 2.4 Let $X$ be one of $l_{1}, L_{1}[0,1], c_{0}, l_{\infty}, L_{\infty}[0,1]$, or $C[0,1]$. Then there exists bounded subsets $S$ in $X$ and elements $f \in \overline{\operatorname{coS}}$ such that the convergence of optimal approximates (convex or linear) to $f \in \overline{\operatorname{coS}}$ is arbitrarily slow.

Proof: Let $\left(a_{n}\right)$ be a sequence converging to 0 that denotes the desired convergence rate. The results then follow immediately by application of Theorem 2.3 with appropriate choice of either $l_{1}$ or $c_{0}$ and of the isomorphism $T$. For $l_{1}$ and $c_{0}$ one obviously takes $T$ to be the identity map. For $L_{1}[0,1]$, first let $g_{n}=2^{n} 1_{\left(2^{-n}, 2^{-n+1}\right)}$ for $n \in \mathbb{N}$, where $1_{(a, b)}$ denotes the characteristic function of the interval $(a, b)$, and define the isometric isomorphism $T$ from $l_{1}$ into $L_{1}[0,1]$ by

$$
\left(a_{1}, a_{2}, \ldots\right) \mapsto \sum_{n=1}^{\infty} a_{n} g_{n}
$$

The remaining examples can all be realized with $T$ mapping from $c_{0}$. Of course, $c_{0} \subset l_{\infty}$, so in that instance we can take $T$ to be the inclusion map. (Alternately one may use the previously described isometric embedding of $l_{1}$ into $l_{\infty}$.) For $C[0,1]$, consider any sequence $g_{1}, g_{2}, \ldots \subset C[0,1]$ where for each $n \in \mathbb{N},\left\|g_{n}\right\|=1$ and $g_{n}(x)=0$ if $x \notin\left(2^{-n}, 2^{-n+1}\right)$. Then define $T$ from $c_{0}$ to $C[0,1]$ via (4). Since $C[0,1] \subset L_{\infty}[0,1]$, this $T$ maps $c_{0}$ into $L_{\infty}[0,1]$ as well.

Theorem 2.3 can be broadly used to identify spaces for which no rate bound is possible, because there are numerous known results characterizing Banach spaces containing subspaces isomorphic to $l_{1}$ or $c_{0}$. For example:

Theorem 2.5 (Bessaga and Pelczynski 1958) Any Banach space that admits an unconditional basis contains a subspace that is either reflexive or is isomorphic to $l_{1}$ or to $c_{0}$. 
Whether or not the preceding theorem was true without the unconditional basis assumption had been an open question until a counter-example was recently produced by W. T. Gowers [10].

Theorem 2.6 (Rosenthal 1974) A Banach space $X$ has a closed subspace isomorphic to $l_{1}$ if and only if every bounded sequence $g_{1}, g_{2}, \ldots$ in $X$ has a subsequence which is weakly Cauchy.

Theorem 2.7 (Rosenthal 1994) If $X$ is a Banach space such that $Y^{*}$ is weakly sequentially complete for all linear subspaces $Y$ of $X$, then $c_{0}$ embeds in $X$.

\subsection{Bounds for Type $t$ Spaces}

We recall some basic definitions first.

A Rademacher sequence $\left(\epsilon_{i}\right)_{i=1}^{n}$ is a finite sequence of independent zero mean random variables taking values from $\{-1,+1\}$. Given any Banach space $X$, any Rademacher sequence $\left(\epsilon_{i}\right)_{i=1}^{n}$, and any fixed finite sequence $\left(f_{i}\right)_{i=1}^{n}$ of elements of $X$, we can view in a natural manner the expression $\sum_{i=1}^{n} \epsilon_{i} f_{i}$ as a random variable taking values in $X$. With this understanding, the space $X$ is of (Rademacher) type $t$ (with constant $C$ ) if for each Rademacher sequence $\left(\epsilon_{i}\right)$ and each sequence $\left(f_{i}\right)$ it holds that

$$
E\left\|\sum \epsilon_{i} f_{i}\right\|^{t} \leq C \sum\left\|f_{i}\right\|^{t}
$$

Theorem 2.8 Let $X$ be a Banach space of type $t$, where $1 \leq t \leq 2$. Pick $S \subset X, f \in \overline{\operatorname{co}(S)}$, and $K>0$ such that $\forall g \in S,\|g-f\| \leq K$. Then for all $n$,

$$
\left\|\operatorname{co}_{n} S-f\right\| \leq \frac{K C^{1 / t}}{n^{1-1 / t}},
$$

where $C$ is a constant depending on $X$ but independent of $n$.

Proof: $\forall \epsilon>0, \exists n_{\epsilon}, \alpha_{1}, \ldots, \alpha_{n_{\epsilon}} \in \mathbb{R}^{+}$, and $f_{1}, \ldots, f_{n_{\epsilon}} \in S$ such that

$$
\begin{aligned}
\sum_{i=0}^{n_{\epsilon}} \alpha_{i} & =1, \\
\sum_{i=0}^{n_{\epsilon}} \alpha_{i} f_{i}+\Delta & =f,
\end{aligned}
$$

and $\|\Delta\|<\epsilon$. Take $\xi_{j}$ to be a sequence of independent random variables on $X$ taking value $f_{i}$ with probability $\alpha_{i}$. Then for any $\beta \in(0,1)$,

$$
E\left\|f-\frac{1}{n} \sum_{j=1}^{n} \xi_{j}\right\|^{t}
$$




$$
\begin{aligned}
& =\frac{1}{n^{t}} E\left\|\sum_{j=1}^{n}\left(f-\xi_{j}\right)\right\|^{t} \\
& =\frac{1}{n^{t}} E\left\|\sum_{j=1}^{n}\left(f-\xi_{j}-\Delta\right)+n \Delta\right\|^{t} \\
& =\frac{1}{n^{t}} E\left((1-\beta) \frac{\left\|\sum_{j=1}^{n}\left(f-\xi_{j}-\Delta\right)\right\|}{1-\beta}+\beta n \frac{\|\Delta\|}{\beta}\right)^{t} \\
& \leq \frac{1}{n^{t}}\left[(1-\beta) E\left(\frac{\left\|\sum_{j=1}^{n}\left(f-\xi_{j}-\Delta\right)\right\|}{1-\beta}\right)^{t}+\beta\left(\frac{n\|\Delta\|}{\beta}\right)^{t}\right] \\
& =\frac{1}{n^{t}(1-\beta)^{t-1}} E\left\|\sum_{j=1}^{n}\left(f-\xi_{j}-\Delta\right)\right\|^{t}+\frac{1}{\beta^{t-1}}\|\Delta\|^{t},
\end{aligned}
$$

which follows because $\phi(x)=x^{t}$ is a convex function for $1 \leq t \leq 2$. Since the range of $\xi_{j}$ has finitely many values and the space is type $\bar{t}$, by (Ledoux and Talagrand [18], Prop. 9.11, p. 248) it follows that:

$$
E\left\|\sum_{j=1}^{n}\left(f-\xi_{j}-\Delta\right)\right\|^{t} \leq C \sum_{j=1}^{n} E\left\|f-\xi_{j}-\Delta\right\|^{t} .
$$

On the other hand, we have:

$$
\begin{aligned}
E\left\|f-\xi_{1}-\Delta\right\|^{t} & =\sum_{i=1}^{n_{\epsilon}} \alpha_{i}\left\|f-f_{i}-\Delta\right\|^{t} \\
& \leq \sum_{i=1}^{n_{\epsilon}} \alpha_{i}\left(\left\|f-f_{i}\right\|+\|\Delta\|\right)^{t} \\
& <\sum_{i=1}^{n_{\epsilon}} \alpha_{i}(K+\epsilon)^{t} \\
& =(K+\epsilon)^{t} .
\end{aligned}
$$

Without loss of generality, assume $0<\epsilon<1$ and take $\beta=\epsilon$. Then combining (7), (8), and (9),

$$
E\left\|f-\frac{1}{n} \sum_{j=1}^{n} \xi_{j}\right\|^{t}<\frac{C(K+\epsilon)^{t}}{n^{t-1}(1-\epsilon)^{t-1}}+\epsilon .
$$


We conclude that for some realization of the $\xi_{j}$ (labeled $g_{j}$ ) the inequality must hold, i.e.,

$$
\left\|f-\frac{1}{n} \sum_{j=1}^{n} g_{j}\right\|^{t}<\frac{C(K+\epsilon)^{t}}{n^{t-1}(1-\epsilon)^{t-1}}+\epsilon .
$$

Taking the infimum with respect to all $\epsilon>0$ proves the theorem.

We now give a specialization to $L_{p}, 1 \leq p<\infty$. These spaces are of type $t=\min \{p, 2\}$. From (Haagerup [11]) we find that the best value for $C$ in (6) is 1 if $1 \leq p \leq 2$ and $\sqrt{2}[\Gamma((p+1) / 2) / \sqrt{\pi}]^{1 / p}$ if $2<p<\infty$. One may use Stirling's formula to get an asymptotic formula for the latter expression.

Corollary 2.9 Let $X$ be an $L_{p}$ space with $1 \leq p<\infty$. Suppose $S \subset X$, $f \in \overline{\operatorname{coS} S}$, and $K>0$ such that $\forall g \in S,\|g-f\| \leq K$. Then for all $n$,

$$
\left\|\mathrm{co}_{n} S-f\right\| \leq \frac{K C_{p}}{n^{1-1 / t}},
$$

where $t=\min \{p, 2\}$, and $C_{p}=1$ if $1 \leq p \leq 2, C_{p}=\sqrt{2}[\Gamma((p+1) / 2) / \sqrt{\pi}]^{1 / p}$ for $2<p<\infty$. For large $p, C_{p} \sim \sqrt{p / e}$.

\subsection{Tightness of Rate Bounds}

We show that the orders of the rate bounds for $L_{p}$ given in (10) are tight. That is, we give specific examples of $L_{p}$ spaces and subsets $S$ with target functions $f \in \overline{\operatorname{coS}}$ where optimal approximants converge with the order specified by our bounds.

Theorem 2.10 There exists $S \subseteq l_{p}, 1<p<\infty$, and $f \in \overline{\operatorname{coS}}$ such that $\left\|\mathrm{co}_{n} S-f\right\|_{p}=K n^{1 / p-1}$ where $K=\sup _{g \in S}\|f-g\|_{p}$.

Proof: Let $S$ consist of the elements of the canonical basis, i.e.,

$$
S=\{(1,0,0, \ldots),(0,1,0,0, \ldots),(0,0,1,0,0, \ldots), \ldots\} .
$$

Then $f:=0$ is in the closed convex hull of $S$ and $\sup _{g \in S}\|f-g\|_{p}=1$. Let $f_{n}$ be an element of $\operatorname{co}_{n} S$ that is to approximate 0 . So $f_{n}$ is of the form

$$
f_{n}=\sum_{k=1}^{n} a_{k} g_{n_{k}},
$$

where each $g_{n_{k}}$ is an element of $S$, and the $a_{k}$ are non-negative and sum to 1 . Without loss of generality, we may assume the $g_{n_{k}}$ are distinct, since otherwise we would be effectively working in $\operatorname{co}_{m} S$ with $m<n$. Then

$$
\left\|f_{n}-0\right\|^{p}=\sum_{k=1}^{n} a_{k}^{p} .
$$


It is easy to see that the error is minimized by taking the $a_{k}$ 's all equal, namely $\forall k, a_{k}=1 / n$ (Jensen). Therefore

$$
\left\|f_{n}-0\right\| \geq n^{(1-p) / p}=n^{1 / p-1}
$$

Next we show that the $O\left(n^{-1 / 2}\right)$ bound for $L_{p}, 2<p<\infty$, is tight (see Corollary 2.9). We borrow from computer science the notation $\psi(n)=\Omega(\phi(n))$ to mean that there is a constant $C>0$ so that $\psi(n) / \phi(n) \geq C$ for all $n$ large enough. For the purposes of the next result, we say a space $L_{p}$ is admissible if there exists a Rademacher sequence definable on it; for instance, $L_{p}(0,1)$ with the usual Lebesgue measure is one such space.

Proposition 2.11 For any admissible $L_{p}, 2<p<\infty$, there exists a subset $S$ and an $f \in \overline{\operatorname{coS}}$ such that $\left\|\operatorname{co}_{n} S-f\right\|_{p}=\Omega\left(n^{-1 / 2}\right)$.

Proof: Let $\xi_{i}$, i.i.d. on $\{-1,+1\}$, be a Rademacher sequence. Define $S=\left\{\xi_{i}\right\}$, which is a subset of the unit ball in $L_{p}$. Using the upper bound of Khintchine's Inequality (Khintchine [17]; Ledoux and Talagrand [18], Lemma 4.1, p. 91), one can show that $0 \in \overline{\operatorname{coS}}$. Suppose the best approximation of 0 by a convex sum of $n$ elements of $S$ is $\sum_{i=1}^{n} \alpha_{i} \xi_{k(i)}$. Then by the lower bound of Khintchine's Inequality,

$$
\left\|\sum_{i=1}^{n} \alpha_{i} \xi_{k(i)}\right\|_{L_{p}} \geq A_{p} \sqrt{\sum_{i} \alpha_{i}^{2}}=A_{p}\left\|\left(\alpha_{i}\right)\right\|_{l_{2}} .
$$

But we have already given an example in $l_{2}$ (in the proof of Theorem 2.10 ) for which the last term is $\Omega\left(n^{-1 / 2}\right)$.

\section{Incremental Approximants}

We now start the study of incremental approximation schemes. Unlike the situation with optimal approximation, incremental approximations are not guaranteed to even converge. In general, the convergence of incremental schemes appears to be intimately tied to the concept of norm smoothness. In Theorem 3.1 we show that smoothness is equivalent to at least a monotonic decrease of the error, and then in Theorem 3.4 it is proved that uniform smoothness is a sufficient condition to guarantee convergence. (It is possible to construct a smooth space with an $\epsilon$-greedy sequence that does not converge-Appendix D. However, if an $\epsilon$-greedy sequence converges, then it can only converge to the desired target function-Corollary 3.2.)

In Sects. 3.2 and 3.3 we study upper bounds on the rate of convergence for spaces with modulus of smoothness of power type greater than 1 and for spaces of (Rademacher) type $t, 1<t \leq 2$. The $L_{p}$ spaces, $1<p<\infty$, are examples 


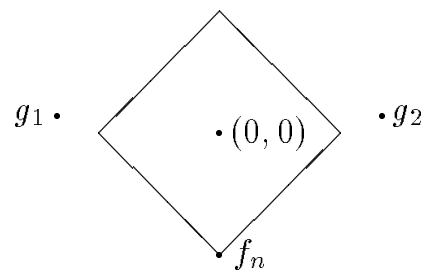

Figure 1: Incremental approximants may fail to converge in some spaces. Consider approximating $f=(0,0)$ by linear combinations of elements from $\left\{f_{n}, g_{1}, g_{2}\right\}$ according to the $L_{1}\left(\mathbb{R}^{2}\right)$ metric. The best approximant by one point is $f_{n}$. The rotated square is the contour of the norm about $f$ on which $f_{n}$ lies. The best approximant by a linear combination of $f_{n}$ and $g_{1}$ or $g_{2}$ is once again $f_{n}$. Thus even though $f$ is in the convex hull of $\left\{f_{n}, g_{1}, g_{2}\right\}$, incremental approximants fail to converge or even to decrease monotonically.

of spaces with modulus of smoothness of power type $t=\min (p, 2)$ (see Appendix B), which themselves sit inside the more general class of spaces of type t. (See, for example, (Lindenstrauss and Tzafriri [22], p. 78; Deville et al. [7], p. 166), while (James [14]) shows that the containment is strict. See also (Figiel and Pisier [9]).) The upper bounds obtained for incremental approximation error in the power type spaces agree with the bounds for optimal approximation error obtained in Sect. 2.2 (albeit with a slightly larger constant of proportionality), which are shown to be the best possible in Sect. 2.3. Therefore little is lost by using incremental approximates instead of optimal approximates, at least in worst-case settings. The incremental convergence bounds obtained in Sect. 3.3 for type- $t$ spaces are weaker, but only slightly, than the optimal approximation error bounds obtained in Sect. 2.2

\subsection{Convergence of Greedy Approximants}

The first remark is that for some spaces there may not exist any nondecreasing rate whatsoever. In the terminology given in the introduction, it may be the case that there are greedy incremental sequences for $f$ for which $\left\|f_{n}-f\right\| \neq$ 0 . This will happen in particular if there are a set $S$ and two elements $f \neq$ $f_{n} \in \operatorname{coS}$ so that for each $g \in S$ and each $h \in \operatorname{co}\left(\left\{f_{n}, g\right\}\right)$ different from $f_{n}$, $\|h-f\|>\left\|f_{n}-f\right\|$; in that case, the successive minimizations result in the sequence $f_{n}, f_{n}, \ldots$, which doesn't converge to $f$. Geometrically, convergence of incremental approximants can fail to occur if the unit ball for the norm has a sharp corner. This is illustrated by the example in Fig. 1, for the plane $\mathbb{R}^{2}$ under the $L^{1}$ norm. In order to use the intuition gained from this example, we need a number of standard but often less-known notions from functional analysis. 
If $X$ is a Banach space and $f \neq 0$ is an element of $X$, a peak functional for $f$ is a bounded linear operator, that is, an element $F \in X^{*}$, that has norm $=1$ and satisfies $F(f)=\|f\|$. (The existence for each $f \neq 0$ of at least one peak functional is guaranteed by the Hahn-Banach Theorem.) Geometrically, one may think of the null space of $F-\|f\|$ as a hyperplane tangent at $f$ to the ball centered at the origin of radius $\|f\|$. (For Hilbert spaces, there is a unique peak functional for each $f$, which can be identified with $(1 /\|f\|) f$ acting by inner products.) The space $X$ is said to be smooth if for each $f$ there is a unique peak functional. (Roughly, this means that balls in $X$ have no "corners.") The modulus of smoothness of any Banach space $X$ is the function $\rho: \mathbb{R}_{\geq 0} \rightarrow \mathbb{R}_{\geq 0}$ defined by

$$
\rho(r):=\frac{1}{2}\left(\sup _{\|f\|=\|g\|=1}\{\|f+r g\|+\|f-r g\|\}-2\right)
$$

Note that, by sub-additivity of norms, always $\rho(r) \leq r$. For Hilbert spaces, one has $\rho(r)=\sqrt{1+r^{2}}-1$. A Banach space is said to be uniformly smooth if $\rho(r)=o(r)$ as $r \rightarrow 0$; in particular, Hilbert spaces are uniformly smooth, but so are $L_{p}$ spaces with $1<p<\infty$, as is reviewed in Appendix B. (Here one has an upper bound of the type $\rho(r)<c r^{t}$, for some $t>1$, which implies uniform smoothness.) As a remark, we point out that uniformly smooth spaces are reflexive, but the converse implication does not hold.

The next result implies that greedy approximants always result in monotonically decreasing error if and only if the space is smooth. In particular, if the space is not smooth, then one can not expect greedy (or even $\epsilon$-greedy) incremental sequences to converge. (Since one may always consider the translate $S-\{f\}$ as well as a translation by $f$ of all elements in a greedy sequence, no generality is lost in taking $f=0$.)

Theorem 3.1 Let $X$ be Banach space. Then:

1. Assume that $X$ is smooth, and pick any $S \subset X$ so that $0 \in \overline{\operatorname{coS}}$. Then for each nonzero $f \in X$ there is some $g \in S$ and some $\tilde{f} \in \operatorname{co}(\{f, g\})$ different from $f$ so that $\|\tilde{f}\|<\|f\|$.

2. Conversely, if $X$ is not smooth, then there exist an $S \subset X$ so that $0 \in \overline{\operatorname{coS}}$ and an $f \in S$ so that, for every $g \in S$ and every $\tilde{f} \in \operatorname{co}(\{f, g\})$ different from $f,\|\tilde{f}\|>\|f\|$.

Proof: Assume that $X$ is smooth, and let $S$ and $f$ be as in the statement. Let $F$ be the (unique) peak functional for $f$. There must be some $g \in S$ for which

$$
F(g)<\|f\| / 2
$$

since otherwise $\{h \in X \mid F(h)=\|f\| / 2\}$ would be a hyperplane separating $\operatorname{co}(S)$ from $0 \in \overline{\operatorname{coS} S}$. Define

$$
f_{\lambda}=(1-\lambda) f+\lambda g \quad \text { for } \lambda \in[0,1] .
$$


We wish to show that $\left\|f_{\lambda}\right\|<\|f\|$ for some $\lambda \in(0,1]$, as this will establish the first part of the Theorem. For this, consider the peak functional $F_{\lambda}$ for $f_{\lambda}$. Note that

$$
\lim _{\lambda \downarrow 0} F_{\lambda}(f)=\lim _{\lambda \downarrow 0} F_{\lambda}\left(f_{\lambda}\right)+F_{\lambda}\left(f-f_{\lambda}\right)=\lim _{\lambda \downarrow 0}\left\|f_{\lambda}\right\|=\|f\| .
$$

The unit ball in $X^{*}$ is weak-* compact (Alaoglu), so the net $\left(F_{\lambda}\right)_{\lambda \in(0,1)}$ (where $\lambda \downarrow 0)$ has a convergent subnet, say $F_{\lambda_{\alpha}} \rightarrow F^{*}$, with $\left\|F^{*}\right\| \leq 1$. The functional $H \mapsto H(f)$ (defined on $H \in X^{*}$ ) is of course continuous with respect to the weak-* topology, so $F_{\lambda_{\alpha}}(f) \rightarrow F^{*}(f)$. By $(12)$ we have $F_{\lambda_{\alpha}}(f) \rightarrow\|f\|$, so $F^{*}$ is in fact a peak functional for $f$. But $X$ is smooth, so $F^{*}=F$. Therefore there exists $\lambda_{0} \in(0,1]$ such that $\left|F_{\lambda_{0}}(g)-F(g)\right|<\|f\| / 2$, which combines with (11) to give $F_{\lambda_{0}}(g)<\|f\|$. Therefore

$$
\begin{aligned}
\left\|f_{\lambda_{0}}\right\| & =F_{\lambda_{0}}\left(f_{\lambda_{0}}\right) \\
& =\left(1-\lambda_{0}\right) F_{\lambda_{0}}(f)+\lambda_{0} F_{\lambda_{0}}(g) \\
& <\|f\|,
\end{aligned}
$$

since $\lambda_{0}>0$. This proves the first assertion.

We now prove the converse. Since $X$ is not smooth, there is some unit vector $f$ with two distinct peak functionals $F$ and $F^{\prime}$, that is, $F$ and $F^{\prime}$ are $\leq 1$ on the unit sphere and $F(f)=F^{\prime}(f)=1$. Since $F \neq F^{\prime}$, there is some $h \in X$ so that $F(h) \neq F^{\prime}(h)$. Let

$$
g:=h-\left(\frac{F(h)+F^{\prime}(h)}{2}\right) f
$$

Note that $F(g)+F^{\prime}(g)=0$ and $F^{\prime}(g) \neq 0$; scaling $g$, we may assume that $F^{\prime}(g)=2$. Consider now the set $S=\left\{f, g_{1}, g_{2}\right\}$, where $g_{1}=g$ and $g_{2}=-g$; note that $0 \in \cos$. This provides the needed counterexample, since

$$
\left\|(1-\lambda) f+\lambda g_{1}\right\| \geq F^{\prime}((1-\lambda) f+\lambda g)=1+\lambda>1=\|f\|
$$

and

$$
\left\|(1-\lambda) f+\lambda g_{2}\right\| \geq F((1-\lambda) f-\lambda g)=1+\lambda>1=\|f\|
$$

for each $\lambda>0$.

It is interesting to remark that, for the set $S$ built in the last part of the proof, even elements in the affine span of $f$ and $g_{1}$ (or of $f$ and $g_{2}$ ) have norm $>1$ if distinct from $f$, that is, the inequalities hold in fact for all $\lambda \neq 0$. (For $\lambda<0$, interchange $F$ and $F^{\prime}$ in the last pair of equations.)

It is an easy consequence of the first part of Theorem 3.1 that greedy incremental approximates in a smooth Banach space can converge only to the target function: 
Corollary 3.2 Let $X$ be a smooth Banach space with $S \subset X$. Let $f \in \overline{\operatorname{coS}}$ and suppose $f_{1}, f_{2}, \ldots$ is an incremental $\epsilon$-greedy sequence with respect to $f$, where the schedule $\epsilon_{1}, \epsilon_{2}, \ldots$ converges to 0 . If the sequence $\left(f_{n}\right)$ converges, then it converges to $f$.

Proof: With out loss of generality, we may assume that $f=0$. Suppose $\lim _{n} f_{n}=f_{\infty} \neq 0$. Then by the first part of Theorem 3.1, there exists $g \in S$ and $\lambda \in[0,1]$ such that $\left\|(1-\lambda) f_{\infty}+\lambda g\right\|<\left\|f_{\infty}\right\|$. Define $\delta=\left\|f_{\infty}\right\|-\left\|(1-\lambda) f_{\infty}+\lambda g\right\|$, and choose $N$ large enough so $\left\|f_{\infty}-f_{n}\right\|<\delta / 3$ and $\epsilon_{n}<\delta / 3$ for all $n>N$. Fix $n>N$. Then $\left\|(1-\lambda) f_{n}+\lambda g\right\|<\left\|f_{\infty}\right\|-2 \delta / 3$, but $\left(f_{n}\right)$ is $\epsilon$-greedy, which implies $\left\|f_{n+1}\right\|<\left\|f_{\infty}\right\|-\delta / 3$. But this is impossible since by choice of $N$, $\left\|f_{\infty}-f_{n+1}\right\|<\delta / 3$. Therefore the limit $f_{\infty}=0$, as desired.

It is possible, however, to have an $\epsilon$-greedy sequence that fails to converge. See Appendix D. This situation is avoided if $X$ is uniformly smooth, as we shall see below. But first we need a technical lemma that captures the geometric properties of smoothness necessary to obtain stepwise estimates of convergence. This lemma is used not only in Theorem 3.4, but also throughout Sect. 3.2.

Lemma 3.3 Let $X$ be a Banach space with modulus of smoothness $\rho(u)$, and let $S \subset X$. Assume that $0 \in \overline{\operatorname{co}(S)}$ and let $f \neq 0$ be an element of $\operatorname{co}(S)$. Let $F$ be a peak functional for $f$. Then

$$
\|(1-\lambda) f+\lambda g\| \leq(1-\lambda)\left[1+2 \rho\left(\frac{\lambda\|g\|}{(1-\lambda)\|f\|}\right)\right]\|f\|+\lambda F(g),
$$

for all $0 \leq \lambda<1$ and all $g \in S$. Furthermore, for any $\epsilon>0$, there exists a $g \in S$ such that $F(g)<\epsilon$.

Proof: Pick any $0 \leq \lambda<1$ and $g \in S$. If $g=0$ then (13) is trivially satisfied, so assume $g \neq 0$.

Define $h=f+u g /\|g\|$ and $h^{*}=f-u g /\|g\|$ for $u \geq 0$. Then from the definition of the modulus of smoothness we have

$$
\|h\|+\left\|h^{*}\right\| \leq 2\|f\|[1+\rho(u /\|f\|)] .
$$

But

$$
\begin{aligned}
\left\|h^{*}\right\| & =\left\|f-\frac{u}{\|g\|} g\right\| \geq F\left(f-\frac{u}{\|g\|} g\right) \\
& =\|f\|-u F(g) /\|g\| .
\end{aligned}
$$

Therefore

$$
\|h\| \leq\|f\|(1+2 \rho(u /\|f\|))+u F(g) /\|g\| .
$$

If we set $u=\lambda\|g\| /(1-\lambda)$, we get

$$
(1-\lambda) f+\lambda g=(1-\lambda) h
$$


which combines with (14) to prove (13).

Finally, given $\epsilon>0$, suppose there is no $g \in S$ such that $F(g)<\epsilon$. Then the affine hyperplane $\{h \in X \mid F(h)=\epsilon / 2\}$ would separate $S$ from 0 , contradicting $0 \in \overline{\operatorname{co}(S)}$.

Theorem 3.4 Let $X$ be a uniformly smooth Banach space. Let $S$ be a bounded subset of $X$ and let $f \in \overline{\operatorname{co}(S)}$ be given, and let $\left(\epsilon_{n}\right)$ be an incremental schedule with $\sum_{k=1}^{\infty} \epsilon_{k}<\infty$. Then any $\epsilon$-greedy (with respect to $f$ ) incremental sequence $\left(f_{n}\right) \subset \cos$ converges to $f$.

Proof: Pick $K \geq \sup _{g \in S}\|f-g\|$, and let $\left(f_{n}\right)$ be an $\epsilon$-greedy incremental sequence. Define

$$
a_{n}:=\left\|f_{n}-f\right\| .
$$

We want to show that $a_{n} \rightarrow 0$. To this end, let $a_{\infty}=\liminf _{n \rightarrow \infty} a_{n}$. Since $\left(f_{n}\right)$ is $\epsilon$-greedy, $a_{n+1} \leq a_{n}+\epsilon_{n}$ and $a_{n+m} \leq a_{n}+\sum_{k=n}^{m-1} \epsilon_{k}$. But $\sum_{k=n}^{\infty} \epsilon_{k} \rightarrow 0$ as $n \rightarrow \infty$, so in fact $a_{\infty}=\lim _{n \rightarrow \infty} a_{n}$. Suppose $a_{\infty}>0$. Then from the definition of $\epsilon$-greedy and (13), it follows that

$$
a_{n+1} \leq \inf _{\lambda, g}\left\{(1-\lambda)\left[1+2 \rho\left(\frac{\lambda K}{(1-\lambda) a_{n}}\right)\right] a_{n}+\lambda F_{n}(g-f)\right\}+\epsilon_{n},
$$

where $\rho$ is the modulus of smoothness for $X, F_{n}$ is the peak functional for $f_{n}-f$, and the infimum is taken over all $0 \leq \lambda<1$ and $g \in S$. (In relation to Lemma 3.3 , everything here is translated by $-f$.) The modulus of smoothness is a non-decreasing function, so certainly

$$
\rho\left(\frac{\lambda K}{(1-\lambda) a_{n}}\right) \leq \rho\left(\frac{2 \lambda K}{(1-\lambda) a_{\infty}}\right)
$$

for large enough $n$. Using this and taking the limit as $n \rightarrow \infty$ in the preceding inequality yields

$$
a_{\infty} \leq a_{\infty}\left\{1+\inf _{\lambda} \lambda\left[\frac{4 K}{a_{\infty}} \frac{\rho(u(\lambda))}{u(\lambda)}-1\right]\right\}
$$

where $u(\lambda):=(2 \lambda K) /\left[(1-\lambda) a_{\infty}\right]$. But $u(\lambda) \rightarrow 0$ as $\lambda \rightarrow 0$, so by uniform smoothness $\rho(u) / u \rightarrow 0$ as $\lambda \rightarrow 0$. Therefore the quantity in the infimum is negative, and a contradiction is reached. Thus, $a_{\infty}$ must be zero.

The stepwise selection of $\lambda=\lambda_{n}$ in the above proof apparently depends upon the modulus of smoothness $\rho(u)$. We shall see in the next section that if we have a non-trivial power type estimate on the modulus of smoothness then it suffices to use $\lambda_{n}=1 /(n+1)$, i.e., $f_{n+1}$ becomes a simple average of $g_{1}, g_{2}, \ldots, g_{n+1}$. 


\subsection{Spaces with Modulus of Smoothness of Power Type Greater than One}

We next give rate bounds for incremental approximates that hold for all Banach spaces with modulus of smoothness of power type greater than one (Theorems 3.5 and 3.7 ). Keep in mind that $\rho(u) \leq \gamma u^{t}$ with $t>1$ is a sufficient condition for $X$ to be uniformly smooth, and holds in particular for $L_{p}$-spaces if $1<p<\infty$. (See Appendix B.)

Theorem 3.5 Let $X$ be a uniformly smooth Banach space having modulus of smoothness $\rho(u) \leq \gamma u^{t}$, with $t>1$. Let $S$ be a bounded subset of $X$ and let $f \in \overline{\operatorname{co}(S)}$ be given. Select $K>0$ such that $\|f-g\| \leq K$ for all $g \in S$, and fix $\epsilon>0$. If the sequences $\left(f_{n}\right) \subset \operatorname{co}(S)$ and $\left(g_{n}\right) \subset S$ are chosen recursively such that

$$
\begin{aligned}
f_{1} & \in S \\
F_{n}\left(g_{n}-f\right) & \leq \frac{2 \gamma}{n^{t-1}\left\|f_{n}-f\right\|^{t-1}}\left((K+\epsilon)^{t}-K^{t}\right):=\delta_{n} \\
f_{n+1} & =\left(\frac{n}{n+1}\right) f_{n}+\left(\frac{1}{n+1}\right) g_{n},
\end{aligned}
$$

(where $F_{n}$ is the peak functional for $f_{n}-f ;$ we terminate the procedure if $f_{n}=$ f), then

$$
\left\|f_{n}-f\right\| \leq \frac{(2 \gamma t)^{1 / t}(K+\epsilon)}{n^{1-\frac{1}{t}}}\left[1+\frac{(t-1) \log _{2} n}{2 t n}\right]^{1 / t}
$$

Recall that (16) can always be obtained, since otherwise $\left\{h \in X \mid F_{n}(h-\right.$ $\left.f)=\delta_{n} / 2\right\}$ would be a hyperplane separating $S$ from $f \in \overline{\operatorname{co}(S)}$.

Proof: Replacing $S$ with $S-f$ allows us to assume without loss of generality that $f=0$ and $\|g\| \leq K$ for all $g \in S$. Also let us write $\tilde{K}$ for $K+\epsilon$.

Applying Lemma 3.3 with $g=g_{n}$ and $\lambda=1 /(n+1)$ yields

$$
\begin{aligned}
\left\|f_{n+1}\right\| & \leq \frac{n\left\|f_{n}\right\|}{n+1}\left[1+2 \rho\left(\frac{\left\|g_{n}\right\|}{n\left\|f_{n}\right\|}\right)\right]+\frac{\delta_{n}}{n+1} \\
& \leq \frac{n\left\|f_{n}\right\|}{n+1}\left[1+\left(\frac{(2 \gamma)^{1 / t} \tilde{K}}{n\left\|f_{n}\right\|}\right)^{t}\right] .
\end{aligned}
$$

If we set

$$
a_{n}:=\frac{n\left\|f_{n}\right\|}{(2 \gamma)^{1 / t} \tilde{K}}
$$

into the previous inequality we obtain

$$
a_{n+1} \leq a_{n}\left(1+1 / a_{n}^{t}\right) .
$$


Applying the triangle inequality to (17) yields

$$
a_{n+1} \leq a_{n}+1 /(2 \gamma)^{1 / t}<a_{n}+3 / 2
$$

by Lemma B.3 (40).

In order to apply Lemma C.3, we need only show that (50) holds for $n=2$ or for $n=1$ with $a_{1} \geq 1$. Note first that

$$
a_{1}=\frac{\left\|f_{1}\right\|}{(2 \gamma)^{1 / t} \tilde{K}}<\frac{1}{(2 \gamma)^{1 / t}}<t^{t}
$$

by Lemma B.3 (43). So (18) holds for $n=1$, and if $a_{1} \geq 1$ then we can apply Lemma C.3 immediately. Otherwise $a_{1}<1$, in which case

$$
a_{2} \leq a_{1}+\frac{1}{(2 \gamma)^{1 / t}}<1+\frac{1}{(2 \gamma)^{1 / t}}<\left(\frac{5 t-1}{2}\right)^{1 / t}
$$

by Lemma B.4, and so (50) holds for $n=2$.

It follows in either case that

$$
a_{n} \leq\left(t n+\frac{t-1}{2} \log _{2} n\right)^{1 / t} \quad \text { for all } n \geq 1 .
$$

Rewriting in terms of $f_{n}$ proves the theorem.

Recall that in $L_{p}$ spaces, the modulus of smoothness is of power order $t$, where $t=\min (p, 2)$. The next corollary follows immediately from the preceding theorem and Lemma B.1.

Corollary 3.6 Let $S$ be a bounded subset of $L_{p}, 1<p<\infty$, with $f \in \overline{\operatorname{co}(S)}$ given. Define $q=p /(p-1)$ and select $K>0$ such that $\|f-g\| \leq K$ for all $g \in S$. Then for each $\epsilon>0$, there exists a sequence $\left(g_{n}\right) \subset S$ such that the sequence $\left(f_{n}\right) \subset \operatorname{co}(S)$ defined by

$$
f_{1}=g_{1} \quad f_{n+1}=n f_{n} /(n+1)+g_{n} /(n+1)
$$

satisfies

$$
\left\|f-f_{n}\right\| \leq \frac{2^{1 / p}(K+\epsilon)}{n^{1 / q}}\left[1+\frac{(p-1) \log _{2} n}{n}\right]^{1 / p}
$$

if $1<p \leq 2$ and

$$
\left\|f-f_{n}\right\| \leq \frac{(2 p-2)^{1 / 2}(K+\epsilon)}{n^{1 / 2}}\left[1+\frac{\log _{2} n}{n}\right]^{1 / 2}
$$

if $2 \leq p<\infty$. 
We now interpret Theorem 3.5 in terms of $\epsilon$-greedy sequences. Let $f, S$, and $X$ be as in that theorem, and let $\left(f_{n}\right)$ be an $\epsilon$-greedy sequence with respect to $f$, which as before we can assume to be 0 . Then

$$
\begin{aligned}
\left\|f_{n+1}\right\| & \leq \inf _{\lambda, g}\left\{(1-\lambda)\left[1+2 \gamma\left(\frac{\lambda\|g\|}{(1-\lambda)\left\|f_{n}\right\|}\right)^{t}\right]\left\|f_{n}\right\|+\lambda F_{n}(g)\right\}+\epsilon_{n} \\
& \leq \inf _{g}\left\{\frac{n\left\|f_{n}\right\|}{n+1}\left[1+2 \gamma\left(\frac{\|g\|}{n\left\|f_{n}\right\|}\right)^{t}\right]+F_{n}(g) /(n+1)\right\}+\epsilon_{n},
\end{aligned}
$$

by Lemma 3.3. The outside inequality holds also if $\left(f_{n}\right)$ is only $\epsilon$-greedy with respect to the convexity schedule $\lambda_{n}=1 /(n+1)$. Now given that the modulus of convexity satisfies $\rho(u) \leq \gamma u^{t}(t>1)$, fix $\epsilon>0$, and select an incremental schedule $\left(\epsilon_{n}\right)$ satisfying $\epsilon_{n} \leq \epsilon \gamma / n^{t}$. Then using the fact that $\|g\| \leq K$ for all $g \in S$ and that there exists $g \in S$ with $F_{n}(g)$ smaller than any preassigned positive value, we get

$$
\left\|f_{n+1}\right\| \leq \frac{n\left\|f_{n}\right\|}{n+1}\left[1+2 \gamma\left(\frac{K}{n\left\|f_{n}\right\|}\right)^{t}\right]+\epsilon \gamma / n^{t}
$$

Recalling the definition of $\delta_{n}$, we see that $\epsilon \gamma / n^{t} \leq \delta_{n} /(n+1)$, and a comparison with (19) shows that the bound obtained in Theorem 3.5 also holds for the $\epsilon$-greedy sequence $\left(f_{n}\right)$ as well. This proves

Theorem 3.7 Let $X$ be a uniformly smooth Banach space with modulus of smoothness $\rho(u) \leq \gamma u^{t}$, with $t>1$. Then $X$ admits incremental convex schemes with rate $1 / n^{1-1 / t}$. Moreover, if the incremental schedule $\left(\epsilon_{n}\right)$ satisfies $\epsilon_{n} \leq$ $\epsilon \gamma / n^{t}$ where $\epsilon$ is any fixed positive value, and if $\left(f_{n}\right)$ is either $\epsilon$-greedy or $\epsilon$ greedy with convexity schedule $\lambda_{n}=1 /(n+1)$, then the error to the target function at step $n+1$ is bounded above by (18).

The specialization of this result to $L_{p}$ spaces, analogous to Corollary 3.6 , is straightforward and is left to the reader.

Remark: The only non-constructive step in the proof of Theorem 3.5 is the determination of $g \in S$ such that $F_{n}(g-f) \leq \delta_{n}$, where $F_{n}$ is the peak functional for $f_{n}-f$. In $L_{p}$ spaces, $F_{n}$ can be associated with the function in $L_{q}(q=p /(p-1))$ defined by

$$
h_{n}(x):=\operatorname{sign}\left(f_{n}-f(x)\right)\left|f_{n}-f(x)\right|^{p-1} /\left\|f_{n}-f\right\|_{p}^{p-1},
$$

So

$$
F_{n}(g-f)=\int h_{n}(x)(g(x)-f(x)) d x .
$$

This means that to satisfy (16), one must find $g \in S$ such that

$$
\int h_{n}(x) g(x) d x \leq \delta_{n}+\int h_{n}(x) f(x) d x .
$$


(We should note that $\int h_{n}(x) f(x) d x$ is likely to be negative.)

The specific details of finding such a $g$ will depend on the neuron class $S$ under consideration. But as an example, suppose $S$ consists of those functions $g(x)$ having the form $\pm \sigma(a \cdot x+b)$, where $a \in \mathbb{R}^{d}, b \in \mathbb{R}, \sigma$ is a fixed activation function, and $x \in \mathbb{R}^{d}$ is allowed to vary over a subset $\Omega \subset \mathbb{R}^{d}$. Then we are left with finding an $a$ and $b$ such that

$$
\left|\int_{\Omega} h_{n}(x) \sigma(a \cdot x+b) d x\right| \geq-\int_{\Omega} h_{n}(x) f(x) d x-\delta_{n} .
$$

Actually, the condition $f \in \overline{\operatorname{coS}}$ implies the existence of an $a$ and $b$ such that the left hand side of (20) is at least as large as $-\int_{\Omega} h_{n}(x) f(x) d x$, so this may be viewed as a maximization problem. We do not need to find the global maximum, however, but only a value satisfying the weaker condition (20).

\subsection{Rate Bounds in Type $t$ Spaces}

We turn our attention now to determining rate bounds for incremental approximants in Rademacher type $t$ spaces with $1<t \leq 2$ (Corollary 3.14). The constants in the bounds are implicit. Furthermore, the rate bounds for the case of $L_{p}$ spaces (not given explicitly) are slightly weaker than those established in the previous section.

Banach and Saks [1] showed that if a sequence $g_{1}, g_{2}, \ldots$ is weakly convergent to $f$ in $L_{p}(0,1), 1<p<\infty$, then there is a subsequence $g_{k_{1}}, g_{k_{2}}, \ldots$ that is Cesaro summable in the norm topology to $f$, i.e., $\left\|f-\sum_{i=1}^{n} g_{k_{i}} / n\right\| \rightarrow 0$. This result was extended to uniformly convex spaces by Kakutani [16]. We give now a generalization that holds in Banach spaces of type $t>1$.

\section{Definition 3.8 (Generalized Banach-Saks Property (GBS))}

A Banach space $X$ has the $G B S$ property if for each bounded set $S$ and each $f \in \overline{\operatorname{coS} S}$, there exists a sequence $g_{1}, g_{2}, \ldots$ in $S$ such that $\left\|f-\sum_{k=1}^{n} g_{k} / n\right\| \rightarrow 0$. If $\phi$ is a given function on $\mathbb{N}$, we say that $X$ has the $G B S(\phi)$ property if for each $f$ and set $S$ as above one can always find some sequence satisfying the convergence rate $\left\|f-\sum_{k=1}^{n} g_{k} / n\right\|=O(\phi(n))$.

A probabilistic proof of the GBS $(\phi)$ property for arbitrary Banach spaces of type $t>1$ is given below. We will make use of the following basic property of type $t$ spaces: If a Banach space $X$ is of type $t$, then for any independent mean zero random variables $\xi_{i} \in X$ taking finitely many values, $E\left(\left\|\xi_{1}+\ldots+\xi_{n}\right\|^{t}\right) \leq$ $C \sum_{i=1}^{n} E\left\|\xi_{i}\right\|^{t}$ (Ledoux and Talagrand [18], p. 248). We also need the following result from (Ledoux and Talagrand [18], Theorem 6.20, p. 171).

Theorem 3.9 Suppose that $\xi_{i}$ are independent mean zero random variables in $X, E\left\|\xi_{i}\right\|^{N} \leq \infty, 1 \leq i \leq n, N>1, N \in \mathbb{N}$. Then there is a universal constant 
$K$ such that

$$
E\left\|\sum_{i=1}^{n} \xi_{i}\right\|^{N} \leq\left(K \frac{N}{\log N}\left[E\left\|\sum_{i=1}^{n} \xi_{i}\right\|+\left(E \max _{1 \leq i \leq n}\left\|\xi_{i}\right\|^{N}\right)^{\frac{1}{N}}\right]\right)^{N} .
$$

The following corollary plays a crucial role in our argument.

Corollary 3.10 Suppose that $\left\|\xi_{i}\right\| \leq M$, and Banach space $X$ is of type $t>1$. Then

$$
E\left\|\sum_{i=1}^{n} \xi_{i}\right\|^{N} \leq A_{N} n^{\frac{N}{t}}
$$

Proof:

$$
\begin{aligned}
E\left\|\sum_{i=1}^{n} \xi_{i}\right\|^{N} & \leq\left(K \frac{N}{\log N}\left[E\left(\left\|\sum \xi_{i}\right\|\right)+M\right]\right)^{N} \\
& \leq\left(K \frac{N}{\log N}\left[\left(E\left\|\sum \xi_{i}\right\|^{t}\right)^{\frac{1}{t}}+M\right]\right)^{N} \\
& \leq\left(K \frac{N}{\log N}\left[C^{\frac{1}{t}}\left(\sum_{i=1}^{n} E\left\|\xi_{i}\right\|^{t}\right)^{\frac{1}{t}}+M\right]\right)^{N} \\
& \leq\left(K \frac{N}{\log N}\right)^{N}\left(C^{\frac{1}{t}} M n^{\frac{1}{t}}+M\right)^{N} \\
& \leq A_{N} n^{\frac{N}{t}} .
\end{aligned}
$$

Here we used the inequality

$$
E(|\xi|) \leq\left[E\left(|\xi|^{t}\right)\right]^{\frac{1}{t}}, \quad t \geq 1,
$$

which is a special case of Jensen's inequality.

Below we follow a standard construction using the Borel-Cantelli Lemma. We first recall this classical result:

Lemma 3.11 ((Borel-Cantelli)) If $\sum P\left(A_{n}\right)<\infty$, then $P(B)=0$, where

$$
B=\bigcap_{k \geq 1} \bigcup_{n \geq k} A_{n} .
$$

Note that in the above equation, $B$ is exactly the set of those $x$ for which $x \in A_{n}$ for infinitely many $n$. 
Theorem 3.12 Let us consider a sequence $\xi_{i}$ of independent, bounded, zero mean random variables in a Banach space $X$ of type $t>1$. Then with probability one,

$$
\left\|\frac{1}{n} \sum_{i=1}^{n} \xi_{i}\right\|=o\left(n^{\frac{1}{t}-1+\epsilon}\right)
$$

for any $\epsilon>0$.

Proof: Using Lemma 3.11, we will prove that for any $a>0$ and $\epsilon>0$,

$$
\sum_{n \geq 1} P\left\{\left\|\frac{1}{n} \sum_{i=1}^{n} \xi_{i}\right\| \geq a n^{\frac{1}{t}-1+\epsilon}\right\}<\infty .
$$

By the Chebyshev inequality, $\forall N \in \mathbb{N}$,

$$
\begin{aligned}
P\left\{\left\|\sum_{i=1}^{n} \xi_{i}\right\| \geq \delta\right\} & \leq E\left(\left\|\sum_{i=1}^{n} \xi_{i}\right\|^{N}\right) \delta^{-N} \\
& \leq A_{N} n^{\frac{N}{t}} \delta^{-N} \\
& :=\Gamma(N, n, \delta) .
\end{aligned}
$$

The second inequality follows from Corollary 3.10. So

$$
\Gamma\left(N, n, a n^{\frac{1}{t}+\epsilon}\right)=\frac{A_{N} n^{\frac{N}{t}}}{a^{N} n^{\frac{N}{t}}+N \epsilon}=\frac{A_{N}}{a^{N} n^{N \epsilon}} .
$$

For sufficiently large $N, N \epsilon>1$ and

$$
\sum_{n \geq 1} \frac{A_{N}}{a^{N} n^{N \epsilon}}<\infty .
$$

Thus by Borel-Cantelli,

$$
\forall a>0, \quad P\left\{\exists\left(n_{k}\right), n_{k} \stackrel{k \rightarrow \infty}{\longrightarrow} \infty \text { s.t. }\left\|\frac{1}{n_{k}} \sum_{i=1}^{n_{k}} \xi_{i}\right\| \geq a\left(n_{k}\right)^{\frac{1}{t}-1+\epsilon}\right\}=0 .
$$

Since the union of countably-many zero-measure sets also has zero measure, it follows that for any $\left(a_{l}\right)$ converging to 0 ,

$$
P\left\{\exists l, \exists\left(n_{k}\right), n_{k} \stackrel{k \rightarrow \infty}{\longrightarrow} \infty \text { s.t. }\left\|\frac{1}{n_{k}} \sum_{i=1}^{n_{k}} \xi_{i}\right\| \geq a_{l}\left(n_{k}\right)^{\frac{1}{t}-1+\epsilon}\right\}=0,
$$

which implies that

$$
P\left\{\lim _{n \rightarrow \infty} \frac{\left\|\frac{1}{n} \sum_{i=1}^{n} \xi_{i}\right\|}{n^{\frac{1}{t}-1+\epsilon}}=0\right\}=1
$$


Now everything is ready to investigate the GBS $(\phi)$ property. Suppose that $f \in \overline{\operatorname{coS}} \subset X$. Then for any $\epsilon>0$ there exists a $k(\epsilon)<\infty$ and $g_{i}^{\epsilon} \in S, \alpha_{i}^{\epsilon}>0$ for $1 \leq i \leq k(\epsilon)$ with $\sum_{i=1}^{k(\epsilon)} \alpha_{i}^{\epsilon}=1$ such that

$$
\left\|f-\sum_{i=1}^{k(\epsilon)} \alpha_{i}^{\epsilon} g_{i}^{\epsilon}\right\|<\epsilon .
$$

Define

$$
\tilde{f}_{\epsilon}:=\sum_{i=1}^{k(\epsilon)} \alpha_{i}^{\epsilon} g_{i}^{\epsilon} .
$$

Let us consider a positive sequence $\left(\epsilon_{n}\right)$ such that

$$
\frac{1}{n} \sum_{j=1}^{n} \epsilon_{j} \leq n^{\frac{1}{t}-1}
$$

Also we need a sequence of independent random variables $\xi_{j}$ such that $P\left\{\xi_{j}=\right.$ $\left.g_{i}^{\epsilon_{j}}\right\}=\alpha_{i}^{\epsilon_{j}}$ for each $i, 1 \leq i \leq k\left(\epsilon_{j}\right)$. Then

$$
\begin{aligned}
\left\|\frac{1}{n} \sum_{j=1}^{n} \xi_{j}-f\right\| & =\left\|\frac{1}{n} \sum_{j=1}^{n}\left(\xi_{j}-\tilde{f}_{\epsilon_{j}}\right)-\frac{1}{n} \sum_{j=1}^{n}\left(f-\tilde{f}_{\epsilon_{j}}\right)\right\| \\
& \leq\left\|\frac{1}{n} \sum_{j=1}^{n} \eta_{j}\right\|+n^{\frac{1}{7}-1} .
\end{aligned}
$$

Here $\eta_{j}=\xi_{j}-\tilde{f}_{\epsilon_{j}}$, so $E \eta_{j}=0$. Applying Theorem 3.12 immediately yields:

Theorem 3.13 Any Banach space of type $t, 1<t \leq 2$, has the GBS( $\left.n^{\frac{1}{t}-1+\epsilon}\right)$ property for all $\epsilon>0$.

This can be restated as:

Corollary 3.14 Let $X$ be a Banach space of type $t, 1<t \leq 2$, and let $S$ be a bounded subset of $X$ with $f \in \overline{c o S}$. Then for all $\epsilon>0$, there exists a sequence $\left(g_{i}\right) \subset S$ such that the incremental sequence

$$
f_{n}=\frac{1}{n} \sum_{i=1}^{n} g_{i}=\frac{n-1}{n} f_{n-1}+\frac{1}{n} g_{n}
$$

satisfies

$$
\left\|f-f_{n}\right\|=o\left(n^{\frac{1}{t}-1+\epsilon}\right) .
$$


Remark: The $\operatorname{GBS}(\phi)$ property guarantees for $f \in \overline{\operatorname{coS}}$ and $S$ bounded the existence of $\left(g_{n}\right) \subset S$ such that $\left\|f-\sum_{i=1}^{n} g_{i} / n\right\| \rightarrow 0$. It is not true in general that one may pick all the $g_{n}$ distinct. Consider for example the Hilbert space $\ell_{2}$ (which is of type 2, i.e., $\operatorname{GBS}(1 / \sqrt{n})$ ) and $S=\left(e_{n}\right)$, where $\left(e_{n}\right)$ is an orthonormal basis. Then $\overline{\cos }=\sum_{i>0} \alpha_{i} e_{i}$ where $\alpha_{i} \geq 0$ and $\sum \alpha_{i}=1$. But if $e_{n_{k}} \neq e_{n_{l}}$, $\left(n_{k} \neq n_{l}\right)$ then

$$
\frac{1}{N} \sum_{i=1}^{N} e_{n_{i}} \stackrel{\|\cdot\|}{\longrightarrow} 0 .
$$

However, it is possible to give necessary and sufficient conditions for the existence of a sequence of distinct elements $\left(g_{i}\right) \subset S$ as above:

Theorem 3.15 Let $X$ be a Banach space of type $t, 1<t \leq 2, S \subset X, f \in \overline{\operatorname{coS}}$. There exists a sequence $\left(g_{i}\right) \subset S$ where $\forall i \neq j, g_{i} \neq g_{j}$ such that $\left\|\sum g_{i} / n-f\right\| \rightarrow$ 0 iff for all finite $K \subset S, f \in \overline{\operatorname{co}(S \backslash K)}$.

Proof: That $f \in \overline{\operatorname{co}(S \backslash K)}$ for all finite $K$ is sufficient follows from the discussion preceding Theorem 3.13. With this condition holding, we are free to choose the $g_{i}^{\epsilon_{j}}$ to be distinct for all $i$ and $j$.

Necessity follows from considering a single finite-cardinality set $K \subset S$ such that $f \notin \overline{\operatorname{co}(S \backslash K)}$. Assume there exists a sequence of distinct elements $\left(g_{i}\right) \subset S$ such that $\left\|f-\sum_{i=1}^{n} g_{i} / n\right\| \rightarrow 0$. We will construct a sequence in $\operatorname{co}(S \backslash K)$ converging to $f$, which is a contradiction.

Since $|K|<\infty$ there must be an $r>0$ such that $\forall n>r, g_{n} \in S \backslash K$. Let $s>r$. Then

$$
f_{s}:=\frac{1}{s} \sum_{i=1}^{s} g_{i}=\frac{1}{s} \sum_{i=1}^{r} g_{i}+\frac{1}{s} \sum_{i=r+1}^{s} g_{i} .
$$

As $s \rightarrow \infty$, the first sum tends to zero, so the second must tend to $f$. Therefore the sequence

$$
\frac{1}{s-r} \sum_{i=r+1}^{s} g_{i}=\frac{s}{s-r} \frac{1}{s} \sum_{i=r+1}^{s} g_{i}
$$

must also tend to $f$. Each element of this sequence is in $\operatorname{co}(S \backslash K)$.

\section{Additional Assumptions on $S$}

We will now study the effect of imposing additional assumptions on the subset $S$ that allow favorable $L_{2}$-like rate bounds in more general spaces. That is, instead of constraining the whole space $X$, we study additional general assumptions on $S$ that allow better rates. The possible sufficiency conditions that we study are: (1) boundedness by $L_{2},(2)$ classes of indicator functions. This last case is especially interesting in pattern classification applications, and the connections with Vapnik-Chervonenkis dimension-first discovered by Barron in (Barron [3]) - are especially intriguing. 


\section{1 $S$ Bounded in $L_{2}$}

For subsets $S$ that happen to be dominated in $L_{2}$, better rates are available.

Proposition 4.1 Let $D$ be a measure space with $m(D)<\infty$. Given $1 \leq p_{1}<$ $p_{2}<\infty$ and $S \subset L_{p_{2}}(D)$, suppose there exists $h \in L_{p_{2}}(D)$ such that for all $g \in S,|g(x)| \leq h(x)$ for a.e. $x \in D$. Then $\bar{S}_{p_{1}}=\bar{S}_{p_{2}}$, where $\bar{S}_{p}$ denotes the closure of $S$ in $L_{p}(D)$. Moreover, if $\left(g_{n}\right) \subset S$ is convergent in $L_{p_{2}}(D)$ to a function $f$, then $\left\|f-g_{n}\right\|_{p_{1}} \leq m(D)^{1 / p_{1}-1 / p_{2}}\left\|f-g_{n}\right\|_{p_{2}}$ for all $n$.

Proof: Since $m(D)<\infty$, we have $S \subset L_{p_{2}}(D) \subset L_{p_{1}}(D)$. Also, $g_{n} \stackrel{p_{1}}{\rightarrow} f$ implies the existence of a subsequence $\left(g_{n_{k}}\right)$ which converges to $f$ a.e.. But both $f$ and the subsequence $\left(g_{n_{k}}\right)$ are bounded pointwise a.e. by $h \in L_{p_{2}}(D)$, so it follows from the dominated convergence theorem that $g_{n_{k}} \stackrel{p_{2}}{\rightarrow} f$. Thus $f \in \bar{S}_{p_{2}}$ and so $\bar{S}_{p_{1}} \subset \bar{S}_{p_{2}}$.

Let $r=p_{2} / p_{1}$ and $s=p_{2} /\left(p_{2}-p_{1}\right)$. Then $r$ and $s$ are conjugate exponents and from Hölder's inequality we have

$$
\int\left|f-g_{n}\right|^{p_{1}} \leq\left\|\left|f-g_{n}\right|^{p_{1}}\right\|_{r}\|1\|_{s}
$$

Taking the $p_{1}$-th root of both sides yields

$$
\begin{aligned}
\left\|f-g_{n}\right\|_{p_{1}} & \leq\left\|f-g_{n}\right\|_{p_{2}}\|1\|_{s p_{1}} \\
& =m(D)^{1 / p_{1}-1 / p_{2}}\left\|f-g_{n}\right\|_{p_{2}},
\end{aligned}
$$

which shows $\bar{S}_{p_{2}} \subset \bar{S}_{p_{1}}$ and provides the stated inequality.

The following Corollary of Theorem 3.5 and Proposition 4.1 shows that for special $S$ we can get $O(1 / \sqrt{n})$ incremental convergence even in $L_{p}$ with $p<2$. (Of course the optimal convergence rate obeys this bound as well.) The result holds in particular for the case where $S$ is a collection of uniformly pointwise bounded functions (for example, indicator functions) on a bounded subset of $\mathbb{R}^{N}$.

Corollary 4.2 Let $S$ be a set of real-valued functions on a finite-measure space $D$ with an $h \in L_{2}(D)$ such that for all $g \in S,|g(x)| \leq h(x)$ for a.e. $x \in D$. Then for any $f \in \overline{\operatorname{coS} S_{p}}, 1 \leq p \leq 2$, there exists an incremental sequence $f_{1}, f_{2}$, ... in $\cos$ with $\left\|f-f_{n}\right\|_{p}=O(1 / \sqrt{n})$.

Proof: The result follows for $p=2$ by Theorem 3.5. For $1 \leq p<2$, take the sequence generated by Theorem 3.5 in $L_{2}(D)$, and apply Proposition 4.1 to show that the $1 / \sqrt{n}$ convergence rate for this sequence holds in $L_{p}(D)$ as well.

Note, however, that in view of Theorem 3.1, we cannot expect every sequence that is $\epsilon$-greedy in $L_{2}(D)$ to be necessarily $\epsilon$-greedy in $L_{p}(D)$. 


\subsection{Results for VC Classes in Sup Norm Spaces}

In Theorem 2.3 it was shown that in general there can be no rate bound in $L_{\infty}$. However, in the special case that $S$ consists of indicator functions and has finite VC dimension, a good rate bound exists even for the sup norm (Theorem 4.6). Our bounds are slightly weaker than that given by (Barron [3]) (ours are "big O" as compared to "little O"). However, the proof method here seems worthy of note, especially as it makes use of more basic results than the central limit theorem relied upon in (Barron [3]).

Next we explore the implications of good convergence rate bounds on the VC dimension of the corresponding set $S$. Good convergence rate bounds for $S$ do not imply that $S$ has a finite $V C$ dimension (Theorem 4.7), i.e., the converse of Theorem 4.6 is false. However, if any nontrivial rate bound holds uniformly for all subsets of $S$, its VC dimension must be finite (Theorem 4.8).

Definition 4.3 Let $F$ be a class of indicator functions on a set $X$. Its dual $F^{\prime}$ is defined as the class $\left\{\mathrm{ev}_{x}, x \in X\right\}$ of indicator functions on $F$, where

$$
\mathrm{ev}_{x}(f)=f(x) \quad \forall f \in F .
$$

Thus, for each element of $X$ there is a unique element of $F^{\prime}$, named $\mathrm{ev}_{x}$. Note that it may be the case that $\mathrm{ev}_{x}=\mathrm{ev}_{y}$ for $x \neq y$. One thinks of $\mathrm{ev}_{x}$ as the "evaluation at $x$ operator" for elements of $F$. Note that $\mathrm{ev}_{x}$ contains the members of $F$ which contain $x$. (If an indicator function takes the value one on an element of its domain it may be said to "contain" it. In fact, we will identify $\mathrm{ev}_{x}$ with $\{f \in F: f(x)=1\}$ )

Definition 4.4 Let $V C$ be the operator on classes of indicator functions that measures the Vapnik-Chervonenkis (VC) dimension. If $F$ is a class of indicator functions, we define the co-VC dimension by

$$
\operatorname{coV} C(F):=\operatorname{VC}\left(F^{\prime}\right)
$$

Lemma 4.5 Denote by $M(\Omega, \Sigma)$ the Banach space of all bounded signed measures $\mu$ on $(\Omega, \Sigma)$ equipped with the norm $\|\mu\|:=|\mu|(\Omega) \equiv \mu^{+}(\Omega)+\mu^{-}(\Omega)$. Let $C \subset \Sigma$; consider the operator $j: M(\Omega, \Sigma) \rightarrow l_{\infty}(C)$ defined by $j(\mu)=(\mu(c))_{c \in C}$. Then $V C(C)<\infty$ iff there exists a constant $K$ such that for any Rademacher sequence $\gamma_{i}$ and all finite sequences $\left(\mu_{i}\right) \subset M(\Omega, \Sigma)$,

$$
E\left\|\sum_{i} \gamma_{i} j\left(\mu_{i}\right)\right\| \leq K\left(\sum_{i}\left\|\mu_{i}\right\|^{2}\right)^{1 / 2} .
$$

If such a $K$ exists, $K=K^{\prime} \sqrt{V C(C)}$, where $K^{\prime}$ is a universal constant.

Proof: Follows trivially from (Ledoux and Talagrand [18], Theorem 14.15, p. 418). 
Theorem 4.6 Let $F$ be a class of indicator functions on a set $X$. Then $F \subset$ $l_{\infty}(X)$. Let $\operatorname{coVC}(F)=d<\infty$. Then for any $h \in \overline{\operatorname{co} F},\left\|\operatorname{co}_{n} F-h\right\| \leq$ $K(d / n)^{1 / 2}$, where $K$ is a universal constant.

Proof: Since $h \in \overline{\operatorname{co} F}, \forall \epsilon>0, \exists k_{\epsilon} \in \operatorname{co} F$ s.t. $\left\|h-k_{\epsilon}\right\|<\epsilon$. We will neglect the $\epsilon$ and write merely $k$ where convenient. For some $n_{\epsilon}, k_{\epsilon}=\sum_{i=1}^{n_{\epsilon}} \alpha_{i} f_{i}$, where $\sum_{i=1}^{n_{\epsilon}} \alpha_{i}=1, a_{i}>0$, and $f_{i} \in F$. Let $\Sigma_{k}:=\sigma\left(F^{\prime} \cup \cup_{i=1}^{n_{\epsilon}}\left\{\left\{f_{i}\right\}\right\}\right) . M\left(F, \Sigma_{k}\right)$ is a Banach space of bounded measures on $F$. The norm of $\mu \in M\left(F, \Sigma_{k}\right)$ is $\|\mu\|_{M}=$ $|\mu|(F)=\mu^{+}(F)+\mu^{-}(F)$. Define $m_{i} \in M\left(F, \Sigma_{k}\right)$ to be the probabilistic pointmass measure with support on $f_{i}$, i.e., $m_{i}$ assigns measure 1 to sets containing $f_{i}$ and 0 otherwise. Define $\mu_{k}:=\sum_{i=1}^{n} \alpha_{i} m_{i}$. Let $\xi_{l}$ be finite-valued i.i.d. random variables taking value $m_{i}$ with probability $\alpha_{i}$. Define $j: M\left(F, \Sigma_{k}\right) \rightarrow l_{\infty}(X)$ by $j(\mu):=\left(\mu\left(\mathrm{ev}_{x}\right)\right)_{x \in X}$. Note that $j$ is a linear operator. By the triangle inequality,

$$
E_{\xi}\left\|j\left(\sum_{i=1}^{n} \xi_{l} / n\right)-h\right\| \leq E_{\xi}\left\|j\left(\sum_{l}\left(\xi_{l}-\mu_{k}\right)\right)\right\| / n+\|h-k\| .
$$

By a simple inequality (Ledoux and Talagrand, [18], Lemma 6.3, p. 152),

$$
E_{\xi}\left\|\sum_{l} j\left(\xi_{l}-\mu_{k}\right)\right\| \leq 2 E_{\xi} E_{\gamma}\left\|\sum_{l} \gamma_{l} j\left(\xi_{l}-\mu_{k}\right)\right\|,
$$

where $\gamma_{l}$ are i.i.d. random variables taking values +1 and -1 with equal probability. Then by Lemma 4.5,

$$
E_{\gamma}\left\|\sum_{l} \gamma_{l} j\left(\xi_{l}-\mu_{k}\right)\right\| \leq K \sqrt{d} \sqrt{\sum_{l}\left\|\xi_{l}-\mu_{k}\right\|_{M}^{2}}
$$

Since $\xi_{l}$ and $\mu_{k}$ are both probability measures, $\left\|\xi_{l}-\mu_{k}\right\|_{M} \leq\left\|\xi_{l}\right\|_{M}+\left\|\mu_{k}\right\|_{M}=2$. Combining the above, we have

$$
E_{\xi}\left\|j\left(\sum_{i=1}^{n} \xi_{l} / n\right)-h\right\| \leq \frac{4 K \sqrt{d}}{\sqrt{n}}+\epsilon .
$$

Since the inequality is true for all $\epsilon>0$ it remains true for $\epsilon=0$. Since for some realization of the $\xi_{l}$ the inequality must still hold, the theorem is proven.

The mere fact of the existence of a convergence rate bound for a set of indicator functions does not imply that the set has finite $\mathrm{VC}$ dimension. We give an example to make this point.

Theorem 4.7 Let $X$ be a measure space with an infinite number of measurable sets. Let $S$ denote the set of characteristic functions of all measurable sets. Clearly the VC dimension of $S$ is infinite. However, if $f \in \overline{\operatorname{coS}}$, then $f$ can be approximated by an element of $\operatorname{co}_{n} S$ with error less than $1 / n$ (in the uniform metric). 
Proof: If $f \in \overline{\operatorname{coS} S}$, then clearly $0 \leq f(x) \leq 1$ for all $x \in X$. Fix $n$ and define

$$
A_{k}=f^{-1}([(k-1) / n, 1])
$$

for $k=1,2, \ldots, n$. (Note that some $A_{k}$ may be empty.) Let $g_{k}$ be the characteristic function for $A_{k}$. Each $g_{k}$ is in $S$ since $f$ must be a measurable function. The function

$$
f_{n}=\sum_{k=1}^{n}(1 / n) g_{k}
$$

is in $\mathrm{co}_{n} S$ and satisfies

$$
0 \leq f_{n}(x)-f(x)<1 / n
$$

for all $x \in X$. (Moreover, this shows that $\overline{\cos }$ equals the set of measurable functions with range in $[0,1]$.)

However, if it is the case that one given convergence rate bound holds for all finite subsets of a set of characteristic functions, then the VC dimension of this set is finite.

Theorem 4.8 Let $F$ be a set of indicator functions on a set $X$ and $C \subseteq F$. Let $\alpha(C, r)$ be the worst-case rate of approximation of a member of the closed convex hull of $C$ by $r$ elements of $C$. Assume that there is some function $h$ so that $h(r) \rightarrow 0$ as $r \rightarrow \infty$ such that $\alpha(C, r) \leq h(r)$ for all finite $C \subset F$. Then $\operatorname{VC}(F)<\infty$.

Proof: We argue by contradiction. Assume that $V C(F)=\infty$. Then also $\operatorname{coV} C(F)=\infty$. Thus, for each integer $n$ there are elements $x_{1}, x_{2}, \ldots, x_{2^{n}} \in X$ and functions $f_{1}, \ldots, f_{n} \in F$ such that $\left(f_{1}, \ldots, f_{n}\right)$ takes all $2^{n}$ possible values on these points. Define $C_{n}:=\left\{f_{1}, \ldots, f_{n}\right\}$. Consider approximating $f:=$ $\sum_{i=1}^{n} f_{i} / n \in \operatorname{co} C_{n}$ by $r$ elements of $C_{n}$. By the symmetry of $f$, we can without loss of generality write the approximant as $g=\sum_{i=1}^{r} \alpha_{i} f_{i}$. Then

$$
\begin{aligned}
\|g-f\|_{\text {sup }} & =\sup _{X}|g(x)-f(x)| \\
& =\sup _{X}\left|\sum_{i=1}^{r}\left(\alpha_{i}-\frac{1}{n}\right) f_{i}(x)-\sum_{i=r+1}^{n} \frac{1}{n} f_{i}(x)\right| \\
& =\frac{1}{2} \sup _{X}\left|\sum_{i=1}^{r}\left(\alpha_{i}-\frac{1}{n}\right)\left[2 f_{i}(x)-1\right]-\sum_{i=r+1}^{n} \frac{1}{n}\left[2 f_{i}(x)-1\right]\right| \\
& =\frac{1}{2}\left(\sum_{i=1}^{r}\left|\alpha_{i}-\frac{1}{n}\right|+\sum_{i=r+1}^{n} \frac{1}{n}\right)
\end{aligned}
$$

since there is an element $x \in X$ for which $2 f_{i}(x)-1$ is of the same sign as $\alpha_{i}-1 / n$ for $1 \leq i \leq r$ and is negative one for $i>r$. Thus

$$
\|g-f\|_{\text {sup }} \geq \frac{1}{2} \frac{n-r}{n}=\frac{1}{2}\left(1-\frac{r}{n}\right) .
$$


Since the bounding function for the error, $h$, converges to zero, there exists $q>0$ such that $h(q)<1 / 4$. But the worst-case error approximating elements of $C_{2 q}$ with $q$ functions from this set is greater than $[1-q /(2 q)] / 2=1 / 4$, a contradiction. This establishes the claim.

\section{Acknowledgements}

This work was partially completed while Michael Donahue and Eduardo Sontag were visiting Siemens Corporate Research.

\section{A Implications of the Convexity Assumption}

Constraining $f$ to lie within the convex closure of $S$ (instead of the linear span) effectively reduces the size of the set of approximable functions. Which functions are being left out? For the case of functions on a real interval under the sup norm where $S$ is the set of Heavisides, there is a tidy answer.

Define $F_{v}:=$

$$
\{f:[a, b] \rightarrow \mathbb{R}|\operatorname{Var}(f) \leq v, \forall t \in[a, b],| f(t) \mid \leq v\} .
$$

The following is an elementary theorem (Lick [20], Exercise 22, p. 364).

Lemma A.1 Let $\left(f_{n}\right)$ be a sequence of functions each in $F_{v}$. Then there exists a subsequence converging everywhere to a limit function also in $F_{v}$.

Let $H$ be the set of Heavisides on $\mathbb{R}$, i.e., $H=\{h: \mathbb{R} \rightarrow \mathbb{R} \mid \exists \theta, h=I[\theta, \infty)\}$. Define

$$
\begin{array}{r}
H_{v}:=\{f: \mathbb{R} \rightarrow \mathbb{R} \mid \exists h \in H \text { s.t. } f=v h \text { or } f=-v h \\
\text { or } f=v h^{\prime} \text { or } f=-v h^{\prime} \\
\text { where } \left.\forall t \in[a, b], h^{\prime}(t)=h(-t)\right\} .
\end{array}
$$

Theorem A.2 $F_{v}={\overline{\operatorname{co}\left(H_{v}\right)}}_{\text {sup }}$.

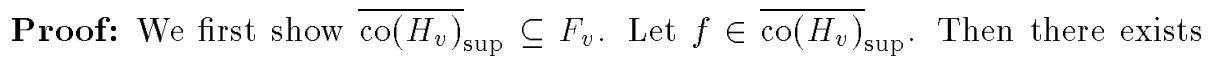
sequence $\left(f_{n}\right)$ with $f_{n} \in \operatorname{co}\left(H_{v}\right)$ and $f_{n} \stackrel{\text { sup }}{\rightarrow} f$. By Lemma A.1, there is a subsequence $\left(f_{k(n)}\right)$ converging everywhere to a limit in $F_{v}$. This limit must be the same function as $f$, so $f \in F_{v}$.

Now we show $F_{v} \subseteq \overline{\operatorname{co}\left(H_{v}\right)_{\text {sup }}}$. Let $f \in F_{v}$, and fix $\epsilon>0$. Since $f$ is of bounded variation, it can have at most a countable number of discontinuities. In particular, if the jump at the $n$th discontinuity is $j_{n}$ then $\sum\left|j_{n}\right| \leq v$, and so $\left|j_{n}\right|<\epsilon$ for all but at most a finite number of $n$ 's. Let $I_{1}, \ldots, I_{k}$ be a partition of $[a, b]$ into finitely many non-intersecting sets that are either intervals or isolated 
points, with $\cup I_{n}=[a, b]$ such that the variation of $f$ on $I_{n}$ is less than $\epsilon$ for all $n$. In constructing such a partition, one makes sure that the opposing sides of jumps of size $\epsilon$ or greater (at most finite in number) belong to different $I_{n}$. Consider a function $f_{\epsilon}$ which is constant on each of the $I_{n}$ with a value no less than the minimum of $f$ on $I_{n}$ and no greater than the maximum of $f$ on $I_{n}$. It is immediately apparent that $f_{\epsilon} \in F_{v}$ and $\left\|f-f_{\epsilon}\right\|_{\text {sup }}<\epsilon$. It is also clear that $f_{\epsilon} \in \operatorname{co}\left(H_{v}\right)$, and since $\epsilon$ was arbitrary, we have established that $f \in \overline{\operatorname{co}\left(H_{v}\right)}$ sup.

By an elementary argument (analogous to the proof of Theorem 4.7), one can show that if $f \in F_{v},\left\|\mathrm{co}_{n} H_{v}-f\right\|_{\text {sup }}=O(1 / n)$.

At this point, it is natural to ask what is the class of functions that can be uniformly approximated by neural nets with Heaviside activations, that is, what is the closure of the linear span (not the convex hull) of the maps from $H_{v}$ (which is the same for all $v$ of course). This is a classical question; see for instance (Dieudonné [8], VII.6): the closure is the set of all regulated functions, that is, the set of functions $f:[a, b] \rightarrow R$ for which $\lim _{x \rightarrow x_{0}^{-}} f(x)$ and $\lim _{x \rightarrow x_{0}^{+}} f(x)$ exist for all $x_{0} \in[a, b)$ and $x_{0} \in(a, b]$, respectively. Thus by constraining target functions to the convex closure of $H_{v}$ instead of the span, we are losing the ability to approximate those regulated functions that are not of bounded variation.

In the multivariable case, that is, $f: K \rightarrow \mathbb{R}$ with $K$ a compact subset of $\mathbb{R}^{n}$, the situation is far less clear. If $f$ has "bounded variation with respect to half-spaces" (i.e., is in the convex hull of the set of all half spaces (Barron [3]), and in particular if $f$ admits a Fourier representation

$$
f(x)=\int_{\mathbb{R}^{n}} e^{i\langle\omega, x\rangle} \tilde{f}(\omega) d \omega
$$

with

$$
\int_{\mathbb{R}^{n}}|| \omega|\| \tilde{f}(\omega)| d \omega<\infty
$$

then by (Barron [3]) there are approximations with rate $O(1 / \sqrt{n}$ ) (since $f$ is in the convex hull of the Heavisides). But the precise analog of regulated functions is less obvious. One might expect that piecewise constant functions can be uniformly approximated, for instance, at least if defined on polyhedral partitions, but this is false.

For a counterexample, let $f$ be the characteristic function of the square $[-1,1]^{2}$ in $\mathbb{R}^{2}$, and let $K$ be, for instance, a disc of radius 2 centered on $(0,0)$. Then it is impossible to approximate $f$ to within error $1 / 8$ by a one hiddenlayer neural net, that is, a linear combination of terms of the form $H(\langle w, x\rangle+b)$, with $w \in \mathbb{R}^{2}$ and $b \in \mathbb{R}$. (Constant terms on $K$ can be included, without loss of generality, by choosing $b$ appropriately.) This is proved as follows. If there would exist a function $g$ of this form, that approximates to within $1 / 8$, then close 
to the boundary of the disc its values are in the range $(-1 / 8,1 / 8)$, and near the center of the square it has values $>7 / 8$. Moreover, everywhere the values of $g$ are in $(7 / 8,+\infty) \bigcup(-1 / 8,1 / 8)$. Now, the function $3 g-(1 / 2)$ is again in the same span, and it now has values in $(2,+\infty)$ and $(-1,0)$ in the same regions. This contradicts (Sontag [27], Prop. 3.8). (Of course, one can also prove this directly.)

\section{B Properties of the Modulus of Smoothness}

In this section we collect some inequalities related to power type estimates of the modulus of smoothness. In particular, the first lemma shows that $L_{p}$ spaces are of power type $t=\min (p, 2)$. (See Corollary 3.6 and Theorem 3.7.)

Lemma B.1 If $X=L_{p}, 1 \leq p<\infty$, then the modulus of smoothness $\rho(u)$ satisfies

$$
\rho(u) \leq \begin{cases}u^{p} / p & \text { if } 1 \leq p \leq 2 \\ (p-1) u^{2} / 2 & \text { if } 2 \leq p<\infty\end{cases}
$$

for all $u \geq 0$.

Proof: From the definition of the modulus of smoothness we have

$$
\begin{aligned}
2(\rho(u)+1) & =\sup \{\|f+g\|+\|f-g\| \mid\|f\|=1,\|g\|=u\} \\
& \leq 2^{1 / q} \sup \left\{\left(\|f+g\|^{p}+\|f-g\|^{p}\right)^{1 / p} \mid\|f\|=1,\|g\|=u\right\},
\end{aligned}
$$

where $q=p /(p-1)$. The inequality follows from the concavity of the function $t \rightarrow t^{1 / p}$. Next we make use of some inequalities given by Hanner [12]:

$$
\begin{aligned}
(\|f\|+\|g\|)^{p}+|\|f\|-\|g\||^{p} & \leq\|f+g\|^{p}+\|f-g\|^{p} \\
& \leq 2\left(\|f\|^{p}+\|g\|^{p}\right),
\end{aligned}
$$

for $1<p \leq 2$. The inequalities hold in the reverse sense if $2 \leq p<\infty$. (The second inequality above is actually due to Clarkson [5].) Combining this with (33) yields

$$
2(\rho(u)+1) \leq \begin{cases}2\left(1+u^{p}\right)^{1 / p} & \text { if } 1 \leq p \leq 2 \\ 2^{1 / q}\left((1+u)^{p}+|1-u|^{p}\right)^{1 / p} & \text { if } 2 \leq p<\infty .\end{cases}
$$

Therefore

$$
\rho(u) \leq \begin{cases}\left(1+u^{p}\right)^{1 / p}-1 & \text { if } 1 \leq p \leq 2 \\ {\left[\frac{(1+u)^{p}+|1-u|^{p}}{2}\right]^{1 / p}-1} & \text { if } 2 \leq p<\infty\end{cases}
$$

This result is cited in (Lindenstrauss [21]). It is possible to use the methods in (Hanner [12]) to show that the above bounds on $\rho(u)$ are tight. 
For $1<p \leq 2$, (32) now follows immediately by Lemma C.1. For $p \geq 2$ and $0 \leq u \leq 1$ a Taylor series expansion provides

$$
\left(\frac{(1+u)^{p}+(1-u)^{p}}{2}\right)^{1 / p}=1+\frac{p-1}{2} u^{2}-\frac{(p-1)(2 p-3)(p+1)}{24} \xi^{4},
$$

for some $\xi \in(0, u)$. In particular, for $p \geq 2$ the last term is negative, and so

$$
\left(\frac{(1+u)^{p}+(1-u)^{p}}{2}\right)^{1 / p}-1 \leq \frac{p-1}{2} u^{2} .
$$

For $u>1$ we divide by $u$ and use (36) again:

$$
\begin{aligned}
\left(\frac{(u+1)^{p}+(u-1)^{p}}{2}\right)^{1 / p} & =u\left(\frac{(1+1 / u)^{p}+(1-1 / u)^{p}}{2}\right)^{1 / p} \\
& \leq u+\frac{p-1}{2 u} .
\end{aligned}
$$

Let $F(u)=1+(p-1) u^{2} / 2-u-(p-1) /(2 u)$. Note that $F(1)=0$ and

$$
\begin{aligned}
F^{\prime}(u) & =(p-1) u-1+(p-1) /\left(2 u^{2}\right) \\
& \geq(p-1)-1 \\
& \geq 0
\end{aligned}
$$

for $u \geq 1$ and $p \geq 2$, so $u+(p-1) /(2 u) \leq 1+(p-1) u^{2} / 2$. Therefore

$$
\left(\frac{(1+u)^{p}+|1-u|^{p}}{2}\right)^{1 / p}-1 \leq \frac{p-1}{2} u^{2}
$$

for all $p \geq 2$ and $u \geq 0$.

For completeness we include the following result.

Theorem B.2 Let $X$ be a Banach space. The modulus of smoothness for $X$ satisfies

$$
\rho(u) \leq u \quad \text { for all } u \geq 0 .
$$

Furthermore, if $X$ is $L_{1}$ or $L_{\infty}$ with dimension at least 2 , then

$$
\rho(u)=u \quad \text { for all } u \geq 0 .
$$

Proof: For an arbitrary Banach space

$$
\begin{aligned}
\rho(u) & =\sup \left\{\frac{\|f+g\|+\|f-g\|}{2}-1 \mid\|f\|=1,\|g\|=u\right\} \\
& \leq \sup \{\|f\|+\|g\|-1 \mid\|f\|=1,\|g\|=u\} \\
& =u
\end{aligned}
$$


proving (37). To prove (38), it evidently suffices to show the existence of $f$ and $g$ with $\|f\|=1$ and $\|g\|=u$ such that $\|f+g\|+\|f-g\|=2(1+u)$. To this end, for $h \in X$ define

$$
h_{>\alpha}(x)=\left\{\begin{array}{cl}
h(x) & \text { if } h(x)>\alpha \\
0 & \text { otherwise }
\end{array}\right.
$$

and

$$
h_{<\alpha}(x)=\left\{\begin{array}{cl}
h(x) & \text { if } h(x)<\alpha \\
0 & \text { otherwise. }
\end{array}\right.
$$

Note that both $h_{>\alpha}$ and $h_{<\alpha}$ are in $X$ for all $\alpha$.

Since $\operatorname{dim}(X) \geq 2$, there must exist a non-constant $h \in X$. For such an $h$ one can always find an $\alpha$ such that $\left\|h_{>\alpha}\right\|>0$ and $\left\|h_{<\alpha}\right\|>0$. If $X=L_{1}$ take

$$
f=\frac{h_{>\alpha}}{\left\|h_{>\alpha}\right\|} \quad g=u \frac{h_{<\alpha}}{\left\|h_{<\alpha}\right\|}
$$

else if $X=L_{\infty}$ select

$$
f=1 \quad g(x)=u[\operatorname{sign}(h(x)-\alpha)] .
$$

In either case $\|f+g\|=\|f-g\|=1+u$, and the result follows.

The following technical lemma uses an inequality of Lindenstrauss to provide several lower bounds on $\gamma$ as a function of $t$ for spaces with modulus of smoothness $\rho(u) \leq \gamma u^{t}$. These are needed in Theorem 3.5.

Lemma B.3 Let $X$ be a Banach space with modulus of smoothness $\rho(u)$ satisfying $\rho(u) \leq \gamma u^{t}$ for all $u \geq 0$. Then $1 \leq t \leq 2$ and

$$
\gamma t \geq\left\{\begin{array}{cl}
{[(2-t) t]^{1-t / 2}(t-1)^{t-1}} & \text { if } 1<t<2 \\
1 & \text { if } t=1 \text { or } t=2 .
\end{array}\right.
$$

Moreover, for all $t \in[1,2]$,

$$
\begin{aligned}
& \gamma \geq \sqrt{2}-1 \\
& \gamma \geq 3^{-t / 2} \\
& \gamma \geq 2^{t-1} / 5^{t / 2}, \\
& \gamma>\frac{1}{t} e^{-3 / 2 e} .
\end{aligned}
$$

Proof: Lindenstrauss [21] gives

$$
\sqrt{1+u^{2}}-1 \leq \rho(u) \leq \gamma u^{t} \quad \text { for all } u \geq 0 .
$$


Letting $u \rightarrow \infty$ shows that $t \geq 1$, and if $t=1$ then $\gamma \geq 1$. Applying the first inequality of Lemma C.1 to $\sqrt{1+u^{2}}$ shows

$$
\frac{u^{2}}{2+u^{2}} \leq \gamma u^{t}
$$

Letting $u \downarrow 0$ proves that $t \leq 2$ and if $t=2$ then $\gamma \geq 1 / 2$.

Therefore $t$ satisfies $1 \leq t \leq 2$, and inequalities (39) through (43) hold if $t=1$ or $t=2$. Next assume $1<t<2$, and rewrite (44) as

$$
\gamma \geq \frac{\sqrt{1+u^{2}}-1}{u^{t}} \quad \text { for all } u \geq 0 .
$$

In particular, this inequality holds if we replace $u$ with $\sqrt{(2-t) t} /(t-1)$, which gives

$$
\gamma \geq \frac{(2-t)^{1-t / 2}(t-1)^{t-1}}{t^{t / 2}}
$$

This completes the proof of (39).

Inequality (40) follows from (44) by setting $u=1$. Using $u=\sqrt{3}$ provides (41), and (42) is obtained with $u=\sqrt{5} / 2$.

To prove (43), place the inequality $x^{x} \geq e^{-1 / e}$ into (45) to obtain

$$
\gamma \geq t^{-t / 2} e^{-1 / 2 e} e^{-1 / e}
$$

Then use $1<t<2$ to complete the proof.

Figure 2 compares these estimates. The relation (39) is the best obtainable from (44). Note in this regard that the $L_{p}$-spaces with $1 \leq p=t \leq 2$ have $\gamma=$ $1 / t$. The remaining inequalities are weaker than (39), but have less complicated forms and are therefore easier to use. The first (40) is a simple bound that is independent of $t$. The inequality (41) is a refinement generally useful for smaller $t$, say $1 \leq t \leq 1.6$, whereas (42) is only slightly improved over the constant estimate (40) for $t$ close to 1 , but significantly better than (41) for $t$ close to 2 . Finally, although (43) is inferior to (41) for all $t$, it has the advantage of showing easily that the product $\gamma t$ is always bigger than $1 / 2\left(e^{-3 / 2 e} \approx 0.576\right)$. Often the form of the estimate is of more importance than its tightness, as can be seen in the proof of Lemma B.4, a technical lemma needed in Theorem 3.5.

Lemma B.4 Let $\rho(u)$ be the modulus of smoothness of a Banach space, and assume that $\rho(u) \leq \gamma u^{t}$ for all $u \geq 0(t \in[1,2]$ is fixed). Then

$$
1+\frac{1}{(2 \gamma)^{1 / t}}<\left(\frac{5 t-1}{2}\right)^{1 / t} .
$$




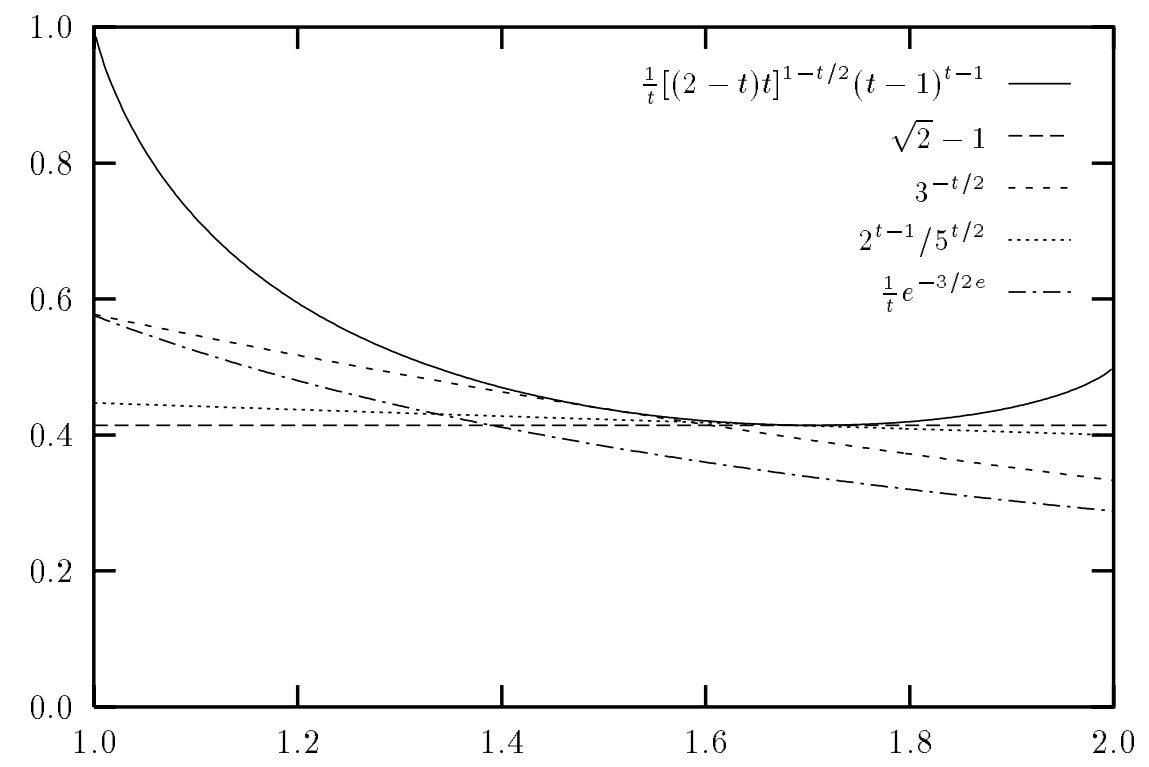

Figure 2: Comparison of lower bound estimates on $\gamma$ (from Lemma B.3) as a function of $t$. 
Proof: Define $h(t)$ to be the right-hand side of (46). Then the derivative of $h(t)$ has the same sign as

$$
\frac{5 t-1}{2}\left(1-\log \left(\frac{5 t-1}{2}\right)\right)+\frac{1}{2}
$$

But (47) is decreasing in $t$ for $t>3 / 5$, so $h^{\prime}(t)=0$ for at most one point $t_{0} \in[1,2]$ (in fact $t_{0} \approx 1.4724$ ), which would be a local maximum for $h$. In particular, for any subinterval $\left[t_{1}, t_{2}\right] \subset[1,2]$, it must be that

$$
h(t) \geq \min \left(h\left(t_{1}\right), h\left(t_{2}\right)\right) \quad \text { for all } t \in\left[t_{1}, t_{2}\right] .
$$

For our purposes we divide the interval $[1,2]$ into two subintervals: $[1,5 / 4]$ and $[5 / 4,2]$. Evaluating $h$ at the endpoints yields

$$
\begin{aligned}
h(1) & =2 \\
h(5 / 4) & =(21 / 8)^{4 / 5}>2.16 \\
h(2) & =3 / \sqrt{2}>2.12 .
\end{aligned}
$$

Therefore $h(t) \geq 2$ for all $t \in[1,5 / 4]$, and $h(t) \geq 2.12$ for all $t \in[5 / 4,2]$.

We now obtain bounds on the left-hand side of (46) on the same intervals via Lemma B.3. From (41) we get

$$
1+\frac{1}{(2 \gamma)^{1 / t}} \leq 1+\frac{\sqrt{3}}{2^{1 / t}} \leq 1+\frac{\sqrt{3}}{2^{4 / 5}}<2 \quad \text { for all } t \in[1,5 / 4] .
$$

Thus (46) holds for $1 \leq t \leq 5 / 4$. Using (42) we obtain

$$
1+\frac{1}{(2 \gamma)^{1 / t}} \leq 1+\frac{\sqrt{5}}{2}<2.12 \quad \text { for all } t \in[5 / 4,2] .
$$

Therefore (46) also holds for $5 / 4 \leq t \leq 2$, and the lemma is proved.

\section{Miscellaneous Inequalities}

Here we collect several inequalities needed in the main body of the text.

Lemma C.1 Let $0 \leq r \leq 1$. Then for all $x>-1$,

$$
\frac{1+x}{1+(1-r) x} \leq(1+x)^{r} \leq 1+r x .
$$

Proof: The right-hand inequality follows from the concavity of the function $(1+x)^{r}$ for $0 \leq r \leq 1$, since $y=1+r x$ is the tangent line to the graph of this function at $x=0$. Applied to $1-r$, this inequality is $(1+x)^{1-r} \leq 1+(1-r) x$, which is the left-hand inequality. 
Lemma C.2 If $1 \leq t \leq 2$, then

$$
(1+x)^{t} \leq 1+t x+t(t-1) x^{2} / 2 \quad \text { for all } x \geq 0 .
$$

Proof: A Taylor series expansion at 0 provides

$$
(1+x)^{t}=1+t x+t(t-1) x^{2} / 2+t(t-1)(t-2) \xi^{3} / 6
$$

for some $\xi \in(0, x)$. The last term is non-positive for $1 \leq t \leq 2$, and the result follows.

The following lemma may be viewed as pertaining to a discrete analogue of the differential equation $y^{\prime}=y^{1-t}$, the general solution of which is $y(x)=$ $(t x+C)^{1 / t}$. The result can be used to provide bounds on the convergence rate of sequences having incremental changes compatible with the estimates from Lemma 3.3. These two results are cobbled together to produce Theorems 3.5 and 3.7 .

Lemma C.3 Let $\left(a_{n}\right)$ be a nonnegative sequence satisfying

$$
a_{n+1} \leq a_{n}+\min \left(\frac{3}{2}, \frac{1}{a_{n}^{t-1}}\right)
$$

for all $n$, where $1 \leq t \leq 2$. If

$$
a_{n} \leq\left(t n+\frac{t-1}{2} \log _{2} n\right)^{1 / t}
$$

is satisfied for some $n \geq 2$, or for $n=1$ with $a_{1} \geq 1$, then it is satisfied for all $n^{\prime}>n$ as well.

Proof: Let us take $b_{n}:=t n+(1 / 2)(t-1) \log _{2} n$, assume that $a_{n} \leq b_{n}^{1 / t}$, and proceed by induction, i.e., show that $a_{n+1} \leq b_{n+1}^{1 / t}$.

Suppose first that $a_{n}<1$ and $n \geq 2$. Then

$$
b_{n+1}^{1 / t} \geq b_{3}^{1 / t} \geq\left(6+(1 / 2) \log _{2} 3\right)^{1 / 2}>5 / 2 .
$$

The second inequality follows from the fact that the function $t \mapsto(3 t+(1 / 2)(t-$ 1) $\left.\log _{2} 3\right)^{1 / t}$ is decreasing in $t$ for $t \in[1,2]$, and so attains its minimum at $t=2$. But then

$$
a_{n+1} \leq a_{n}+3 / 2<5 / 2<b_{n+1}^{1 / t},
$$

as desired.

Alternatively, suppose that $a_{n} \geq 1$. Then since the function $x \mapsto x\left(1+1 / x^{t}\right)$ is nondecreasing for $x \geq 1$, we have

$$
a_{n+1} \leq a_{n}\left(1+1 / a_{n}^{t}\right) \leq b_{n}^{1 / t}\left(1+1 / b_{n}\right)
$$




$$
\begin{aligned}
& \leq b_{n}^{1 / t}\left[1+\frac{t}{b_{n}}+\frac{t(t-1)}{2 b_{n}^{2}}\right]^{1 / t} \quad \text { (by Lemma C.2) } \\
& \leq\left[t(n+1)+\frac{t-1}{2} \log _{2}(n+1)+\frac{t-1}{2 n}-\frac{t-1}{2} \log _{2}(1+1 / n)\right]^{1 / t} .
\end{aligned}
$$

By the concavity of the logarithm, $\log _{2}(1+1 / n) \geq 1 / n$ (for $n \geq 1$ ), which along with the definition of $b_{n+1}$ yields

$$
a_{n+1} \leq b_{n+1}^{1 / t}
$$

concluding the proof.

\section{An Example of a Smooth Banach Space with Non-Converging $\epsilon$-Greedy Sequences}

In Sect. 3.1 it was shown that $\epsilon$-greedy sequences in uniformly smooth spaces always converge (provided $\sum \epsilon_{k}<\infty$ ), and that smoothness is necessary and sufficient for monotonically decreasing error in incremental approximants. We now construct an example showing that simple smoothness is insufficient to guarantee convergence of $\epsilon$-greedy sequences.

Let $a=(a(n))$ be a sequence of real numbers. Define the sequence of functions $\left(F_{n}\right)$ (the norm sequence) from $\mathbb{R}^{\mathbb{N}}$ to $\mathbb{R}^{+} \cup\{0\}$ recursively by

$$
\begin{aligned}
& F_{1}(a)=|a(1)| \\
& F_{n}(a)=\left[\left(F_{n-1}(a)\right)^{p_{n}}+|a(n)|^{p_{n}}\right]^{1 / p_{n}},
\end{aligned}
$$

where $\left(p_{n}\right)$ is a fixed sequence (called the norm power sequence) with $1 \leq p_{n}<$ $\infty$ for all $n$.

Note that for each $a, F_{n}(a)$ is nondecreasing with $n$. Define

$$
X_{\left(p_{n}\right)}:=\left\{a \in \mathbb{R}^{\mathbb{N}} \mid \sup _{n} F_{n}(a)<\infty\right\},
$$

and for $a \in X_{\left(p_{n}\right)}$

$$
\|a\|:=F(a):=\lim _{n \rightarrow \infty} F_{n}(a) .
$$

The reader may verify that $X_{\left(p_{n}\right)}$ equipped with the norm (52) is a Banach space. (This space is similar to the modular sequence spaces studied in (Woo [29]).) If $\left(p_{n}\right)$ is bounded and $p_{n}>1$ for all $n$, then it can be shown that $X_{\left(p_{n}\right)}$ is smooth. Also we use the notation $e_{n} \in X_{\left(p_{n}\right)}$ to denote the canonical basis element $e_{n}(k)=\delta_{n}(k)$. (Note that $\left\|e_{n}\right\|=1$ for all $n$ independent of $\left(p_{n}\right)$.) 
Proposition D.1 Let $\left(\gamma_{n}\right)$ be a strictly decreasing sequence converging to 1 . Then there exists a norm power sequence $\left(p_{n}\right)$ that is non-increasing, converges to 1 , and has $p_{n}>1$ for all $n$, such that the bounded set $S=\left\{-\gamma_{1} e_{1}\right\} \cup$ $\left\{\gamma_{n} e_{n}\right\}_{n \in \mathbb{N}}$ in $X_{\left(p_{n}\right)}$ admits for each incremental schedule $\left(\epsilon_{n}\right)$ an incremental sequence $\left(a_{n}\right) \subset \operatorname{coS}$ that is $\epsilon$-greedy with respect to 0 but does not converge. (Note that $0 \in \operatorname{coS}$.)

Proof: $X_{\left(p_{n}\right)}$ is determined by its power sequence $\left(p_{n}\right)$. Choose $p_{1}>1$ arbitrarily, and recursively select $p_{n} \in\left(1, p_{n-1}\right]$ to satisfy

$$
\inf _{0 \leq \lambda \leq 1}\left[(1-\lambda) \gamma_{n-1}\right]^{p_{n}}+\left(\lambda \gamma_{n}\right)^{p_{n}}>1
$$

This can always be done because the inequality holds for $p_{n}=1$ and so by continuity in $p_{n}$ holds also for all $p_{n}$ sufficiently close to 1 .

Now let $\left(\epsilon_{k}\right)$ be a fixed incremental schedule. We build an $\epsilon$-greedy (with respect to 0$)$ sequence $\left(a_{k}\right) \subset S \subset \operatorname{co} S$ as follows. Let $a_{1}=\gamma_{n_{1}} e_{n_{1}}$ be any $\epsilon_{1}$-greedy element of $S$ with $n_{1}>1$ (i.e., $\gamma_{n_{1}}<\min \left\{1+\epsilon_{1}, \gamma_{1}\right\}$ ). Assuming that

$a_{k-1}=\gamma_{n_{k-1}} e_{n_{k-1}}$ with $n_{k-1}>1$, we will show that we can pick $a_{k}=\gamma_{n_{k}} e_{n_{k}}$ with $n_{k}>n_{k-1}$ to be $\epsilon_{k}$-greedy. Indeed, suppose that

$$
b_{k}=(1-\lambda) a_{k-1}+\lambda g_{k}
$$

is an $\epsilon_{k}$-greedy step, where $g_{k} \in S$. It follows from (53) and the monotonicity of $\gamma_{n}$ that $\left\|b_{k}\right\|>1$, so we can pick $n_{k}>n_{k-1}$ such that

$$
\left\|b_{k}\right\|>\gamma_{n_{k}}=\left\|\gamma_{n_{k}} e_{n_{k}}\right\| \text {. }
$$

Therefore, taking $a_{k}=\gamma_{n_{k}} e_{n_{k}}$ yields an $\epsilon_{k}$ greedy increment.

We define the sequence $\left(a_{k}\right)$ recursively in this manner to complete the construction.

\section{References}

[1] S. BANACH AND S. SAKS, Sur la convergence forte dans les champs $L^{P}$, Studia Math., 2 (1930), pp. 51-57.

[2] A. R. BARRoN, Approximation and estimation bounds for artificial neural networks, in Proc. Fourth Annual Workshop on Computational Learning Theory, M. Kaufmann, 1991, pp. 243-249.

[3] - Neural net approximation, in Proc. of the Seventh Yale Workshop on Adaptive and Learning Systems, 1992, pp. 69-72.

[4] C. Bessaga AND A. PelczYnski, A generalization of results of R. C. James concerning absolute bases in Banach spaces, Studia Math., 17 (1958), pp. 151-164. 
[5] J. A. Clakkson, Uniformly convex spaces, Trans. Amer. Math. Soc., 40 (1936), pp. 396-414.

[6] C. Darken, M. Donahue, L. Gurvits, and E. Sontag, Rate of approximation results motivated by robust neural network learning, in Proceedings of the Sixth Annual ACM Conference on Computational Learning Theory, The Association for Computing Machinery, 1993, pp. 303-309.

[7] R. Deville, G. Godefroy, and V. Zizler, Smoothness and Renormings in Banach Spaces, Wiley, N.Y., 1993.

[8] J. Dieudonné, Foundations of Modern Analysis, Academic Press, N.Y., 1960.

[9] T. Figiel AND G. Pisier, Séries aléatoires dans les espaces uniformément convexes ou uniformément lisses, C. R. Acad. Sci. Paris, 279 (1974), pp. 611-614.

[10] W. T. Gowers, A Banach space not containing $c_{0}, l_{1}$, or a reflexive subspace. Preprint.

[11] U. HaAgerup, The best constants in the Khintchine inequality, Studia Math., 70 (1982), pp. 231-283.

[12] O. Hanner, On the uniform convexity of $L^{p}$ and $\ell^{p}$, Arkiv för Matematik, 3 (1956), pp. 239-244.

[13] S. J. Hanson and D. J. BURR, Neural Information Processing Systems, American Institute of Physics, New York, 1988, ch. Minkowski-r Backpropagation: Learning in Connectionist Models with Non-Euclidean Error Signals, p. 348.

[14] R. C. JAmEs, Nonreflexive spaces of type 2, Israel Journal Math., (1978), pp. $1-13$.

[15] L. K. JonEs, A simple lemma on greedy approximation in Hilbert space and convergence rates for projection pursuit regression and neural network training, The Annals of Statistics, 20 (1992), pp. 608-613.

[16] S. KaKUTANI, Weak convergence in uniformly convex spaces, Tôhoku Math. Journal, 45 (1938), pp. 188-193.

[17] J. Khintchine, Uber die diadischen Brüche, Math. Z., 18 (1923), pp. 109116.

[18] M. Ledoux and M. Talagrand, Probability in Banach Space, SpringerVerlag, Berlin, 1991. 
[19] M. Leshno, V. Lin, A. Pinkus, And S. Schocken, Multilayer feedforward networks with a non-polynomial activation function can approximate any function. Preprint, 1992.

[20] D. R. Lick, The Advanced Calculus of One Variable, Meredith, New York, 1971.

[21] J. Lindenstrauss, On the modulus of smoothness and divergent series in Banach spaces, The Michigan Math. Journal, 10 (1963), pp. 241-252.

[22] - Classical Banach Spaces II: Function Spaces, Springer-Verlag, Berlin, 1979.

[23] M. J. D. Powell, Approximation Theory and Methods, Cambridge University Press, Cambridge, 1981.

[24] W. J. REY, Introduction to Robust and Quasi-Robust Statistical Methods, Springer-Verlag, Berlin, 1983.

[25] H. RosenthaL, A characterization of Banach spaces containing $l_{1}$, in Proc. Nat. Acad. Sci. (USA), vol. 71, 1974, pp. 2411-2413.

[26] - A subsequence principle characterizing Banach spaces containing $c_{0}$, Bull. Amer. Math. Soc., 30 (1994), pp. 227-233.

[27] E. D. SonTAG, Feedback stabilization using two-hidden-layer nets, IEEE Trans. Neural Networks, 3 (1992), pp. 981-990.

[28] K. R. Stromberg, An Introduction to Classical Real Analysis, Wadsworth, New York, 1981.

[29] J. Y. T. Woo, On modular sequence spaces, Studia Math., 48 (1973), pp. $271-289$.

Michael J. Donahue

Institute for Mathematics and its Applications

University of Minnesota

Minneapolis, MN 55455

Christian Darken

Learning Systems Department

Siemens Corporate Research, Inc.

755 College Road East

Princeton, NJ 08540
Leonid Gurvits

Learning Systems Department

Siemens Corporate Research, Inc. 755 College Road East

Princeton. NJ 08540

Eduardo Sontag

Department of Mathematics

Rutgers University

New Brunswick, NJ 08903 